\title{
LE PALÉogène de la COUPE de LA ROUTE GAN-RÉBÉNACQ (AQUITAINE, FRANCE) : STRATIGRAPHIE INTÉGRÉE, FORAMINIFÈRES ET NANNOFOSSILES CALCAIRES
}

\author{
THE PALEOGENE OF THE GAN-RÉBÉNACQ ROAD SECTION \\ (AQUITAINE, FRANCE) : INTEGRATED STRATIGRAPHY, \\ FORAMINIFERA AND CALCAREOUS NANNOFOSSILS
}

\author{
par Étienne STEURBAUT* et Kàroly SZTRÀKOS**
}

\begin{abstract}
Résumé. - La révision de la coupe de la route Gan-Rébénacq a démontré que la succession entre la base du Sélandien jusqu'au milieu de l'Yprésien (60.0 Ma - 50.7 Ma) est relativement complète dans la région de Gan, malgré la présence d'une série de failles. L'étude des nannofossiles calcaires (zones NP) et des foraminifères planctoniques (zones $\mathbf{P}$ ) a permis de préciser l'âge des divers membres de la Formation de Pont-Labau et des Marnes de Gan. La séquence de dépôt sélandienne P6 (NP 4-NP 5 ; P 3b-P 4a), les séquences thanétiennes P7 à P9 (NP 6 à NP 9 ; P 4a-P 4c) et les séquences éocènes basales P10-E2 et P11-E3 (NP 9- ?NP 10 ; P 5), qui composent la partie principale de la Formation de Pont-Labau, ont été reconnues sur le terrain. La signification séquentielle du Membre d'Artigueloutan (NP 11, P 6b) reste à préciser dans la région de Gan. La base sableuse des Marnes de Gan, observée dans la coupe du ruisseau Las Hiès à Gan, se rattache à la base de la partie supérieure de la zone NP 12. L'intégration des données a conduit à la reconstitution de l'histoire sédimentaire de la région de Gan. Celle-ci est marquée par une série de changements du niveau marin, liée à la tectonique pyrénéenne.
\end{abstract}

ABSTRACT. - The revision of the Gan-Rébénacq road section reveals that the succession between the base of the Selandian up to the middle of the Ypresian (60.0 Ma - 50.7 Ma) is rather complete in the Gan area, despite the presence of a series of faults. The age of the different members of the Pont-Labau Formation and of the Gan Marls has been accurately established in terms of calcareous nannofossil (NP zones) and planktonic foraminiferal (P zones) analyses. The Selandian depositional sequence P6 (NP 4-NP 5 ; P 3b-P 4a), the Thanetian sequences P7 to P9 (NP 6 to NP 9; P 4a-P 4c) and the earliest Eocene sequences P10 and P11 (NP 9- ?NP 10; P 5), composing the main part of the Pont-Labau Formation, have been identified in the outerops. The sequence stratigraphical significance of the Artigueloutan Member (NP 11, P 6b) remains to be clarified in the Gan area. The sandy base of the Gan Marls as observed in the "Las Hiès " river section at Gan, has been assigned to the lowermost upper NP 12. Integration of all available data has led to the reconstruction of the depositional history of the Gan area. This history is marked by a series of sea-level changes, which are controlled by the Pyrenean tectonism.

Mots-clés : Nannofossiles calcaires - Foraminifères - Paléogène - Coupe de la route Gan-Rébénacq - Aquitaine - SW France

Key-words : Calcareous nannofossils - Foraminifera - Paleogene - Gan-Rébénacq road section - Aquitaine - SW France

\section{INTRODUCTION}

Célèbres par ses riches faunes malacologiques (O'Gorman, 1923), les marnes de la tuilerie de Gan, ainsi que la série sous-jacente, largement exposée à
Pont-Labau et le long de la route Gan - Rébénacq, $3 \mathrm{~km}$ plus au sud (Fig. 1), figurent dans la plupart des synthèses stratigraphiques classiques sur l'Aquitaine (Douvillé, 1919 ; Gubler et Pomeyrol, 1946 ; Daguin, 1948). Il faut cependant attendre jusqu'en 1967 pour voir apparaître les premières études dé-

* Institut Royal des Sciences Naturelles de Belgique (IRScNB), 29 rue Vautier, B-1000 Bruxelles, Belgique et KULeuven, Belgique ; e-mail : Etienne.Steurbaut@naturalsciences.be ;

** Hall A3, 35 rue Savier, F-92240 Malakoff, France ; e-mail : karoly_sztrakos@hotmail.com 
taillées par Hay et Mohler, et quasi parallèlement, par Lezaud et par Flicoteaux. A partir d'une trentaine d'échantillons collectés le long de la route GanRebenacq (D 934, aussi appelée dans la littérature route de Laruns), entre le lieu-dit Guillempau (feuille topographique 1546 est, Arudy ; $x=378.475, y=$ $3103.500)$ et le carrefour vers Lapeyre (1545 est, Pau ; $x=378.575 ; y=3103.950$ ) (Fig. 2), Hay et Mohler ont reconnu quatre zones à nannofossiles calcaires, codifiées ultérieurement par Martini (1971) en zones NP 5, NP 6, NP 7 et NP 9. Convaincus de la continuité de la série, Hay et Mohler en ont fait une coupe de référence pour le Paleocène supérieur. La lacune d'observation entre les échantillons 826 et 827 fut invoquée pour le non-repérage de la zone à Heliolithus riedelii (= NP 8 de Martini). Les couches affleurant le long de la rivière le Néez (Nez sur les nouvelles cartes topographiques) à Pont-Labau ont été attribuées au Danien par ces mêmes auteurs (présence de la zone à Markalius astroporus $(=M$. inversus) et la zone à Cruciplacolithus tenuis, équivalents des zones NP 1 et NP 2 de Martini), celles de la tuilerie de Gan à l'Yprésien (zone NP 12), confirmant ainsi les conclusions de Martini (1961).

En 1962 Lezaud a présenté une liste d'une dizaine d'espèces de nannofossiles calcaires en provenance de la tuilerie de Gan lors du Colloque sur le Paléogène à Bordeaux (Lezaud, 1964). Plus tard, dans le cadre d'une thèse de doctorat ce même auteur discute 8 échantillons en provenance de la route de Gan-Rébénacq et de la carrière de Pont Labau, mais sans donner des localisations précises (Lezaud, 1967). Dans sa thèse de doctorat Flicoteaux (1967) discute l'ensemble des foraminifères planctoniques et les caractères lithologiques et sédimentologiques du Paléocène des environs de Gan. Une synthèse de ce travail a été présentée en 1970 pendant le colloque sur les méthodes et tendances de la stratigraphie (Flicoteaux, 1972). Kapellos et Schaub (1973) ont étudié les nannofossiles calcaires et les grands foraminifères de plusieurs affleurements des Marnes de Gan. Bigg (1982) a fait l'inventaire micropaléontologique de 5 échantillons de la tuilerie de Gan. Les coupes de Gan-Rébénacq et de la Tuilerie de Gan ont été reprises par Seyve (1984) dans le cadre d'une thèse de doctorat. Cet auteur a observé les zones NP 4, NP 6 et NP 7 aux alentours de l'embranchement du chemin de Guillempau et de la route Gan-Rébénacq.

Vers le milieu des années 80, suite aux travaux d'amélioration des routes en Aquitaine méridionale, la coupe de la route Gan-Rébénacq a regagné l'intérêt des géologues. L'élargissement de la route a mis à jour plusieurs nouvelles coupes et a permis de rafraîchir les anciennes tranchées. Malgré ces conditions

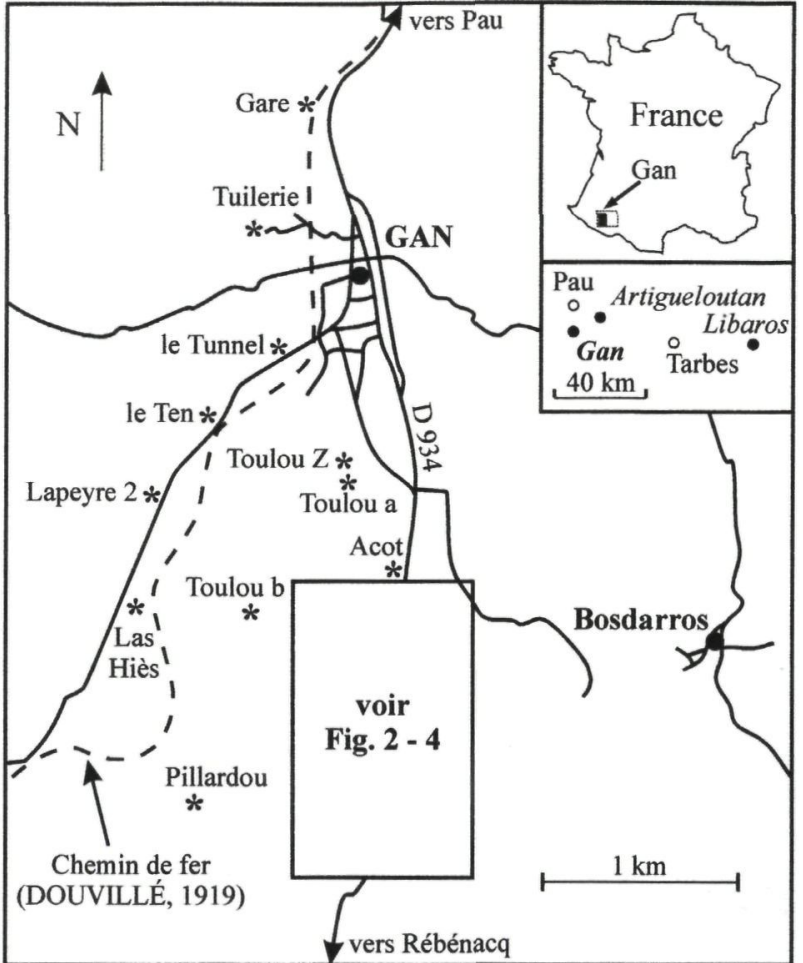

FIG. 1. - Localisation des affleurements étudiés dans la région de Gan.

Location of the studied outcrops in the Gan area.

exquises de terrain et le progrès dans la connaissance de la lithostratigraphie et l'analyse séquentielle du Paléogène sud-aquitain (Cavelier et al., 1997 ; Sztràkos et al., 1997, 1998 ; Fondecave-Wallez et al., 1999 ; Sztràkos, 2000 ; Gély et Sztràkos, 2000), la succession stratigraphique n'a plus été détaillée. Le présent travail est destiné à combler cette lacune en fournissant des levés et des échantillonnages très serrés de la série, afin de situer pour la première fois les unités lithostratigraphiques, définies par Sztràkos et al. (1997), avec précision sur le terrain, et de préciser leurs âges respectifs à partir des analyses micropaléontologiques détaillées. Les coupes présentées ici se localisent entre le lieu-dit Guillempau et la maison Bourbon (1545 est, Pau ; $\mathrm{x}=378.925 ; \mathrm{y}=$ $3104.625), 1.3 \mathrm{~km}$ plus au nord (Fig. 2). Elles ont été levées entre 1993 et 1995. Actuellement, certaines parties de la coupe sont beaucoup moins accessibles, envahies par la végétation. 


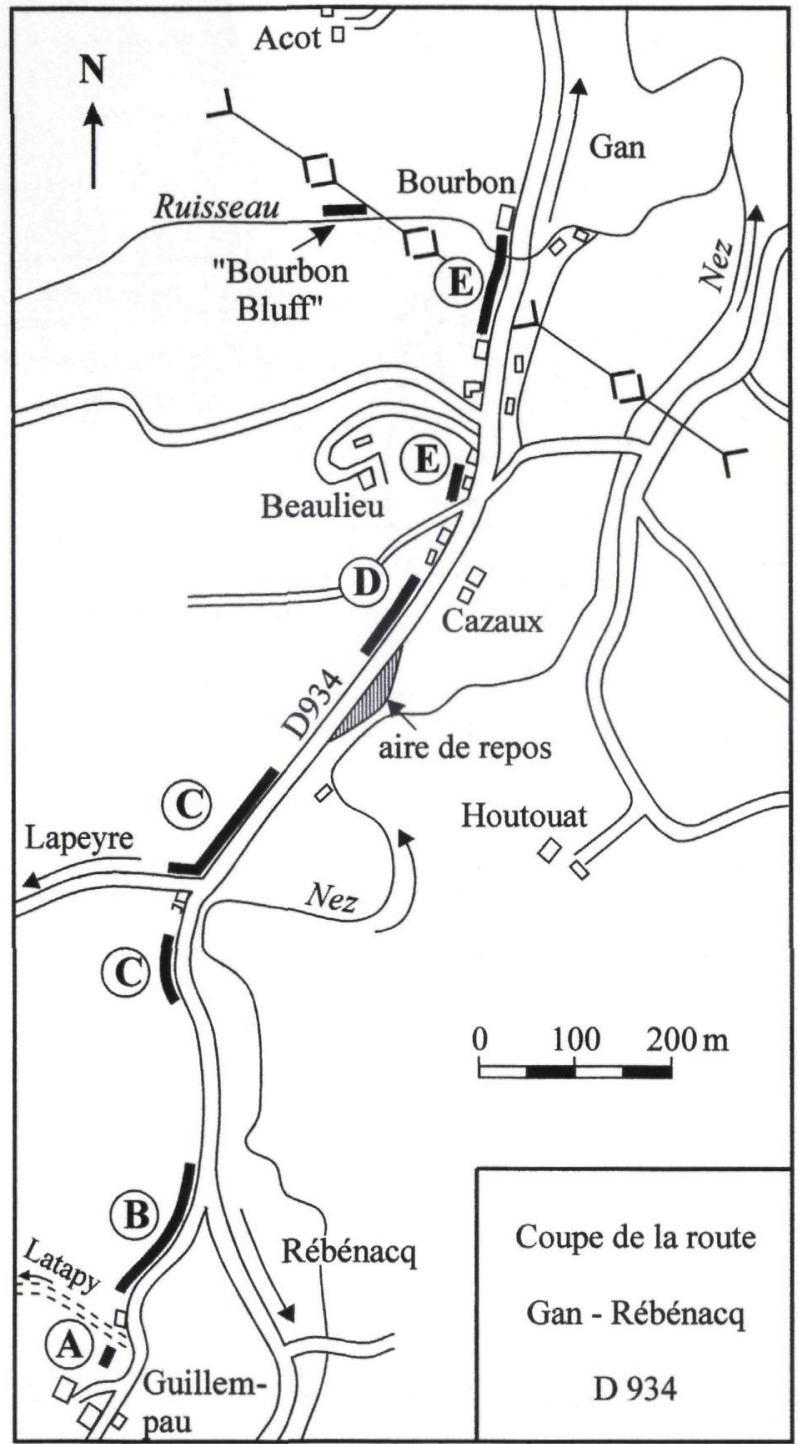

FIG. 2. - La coupe de la route Gan-Rébénacq et localisation des affleurements.

The Gan-Rébénacq road section with location of the outcrops.

\section{LITHOSTRATIGRAPHIE}

La coupe de la route Gan-Rébénacq est le stratotype de la Formation de Pont-Labau de Hay et Mohler (1967), revue par Sztràkos et al. en 1997. Ces derniers ont découpé cette formation en plusieurs membres dans sa localité-type, de bas en haut, les Sables de Guillempau, les Marnes de Latapy, les Couches de Néez, les Couches de Libaros, les Couches du Pillardou et le Marnes d'Artigueloutan. La révision de cette coupe dans le présent travail a permis de reconnaître ces membres sur le terrain. Toutes les mesures citées ci-dessous sont des distances en $\mathbf{m}$ calculées le long des bordures de chemins à partir du point 0 , situé au croisement du cul-de-sac vers Guillempau et d'un petit chemin partant vers le nord au hameau de Latapy (Fig. 2). Comme la direction des chemins varie considérablement à la suite des virages, ces distances ne peuvent pas être confondues avec des épaisseurs. Celles-ci sont généralement beaucoup plus faibles que les mesures le long de chemins.

Les Sables de Guillempau, le membre basal de la Formation de Pont-Labau, sont présents dans la coupe A et jusqu'à $20 \mathrm{~m}$ dans le coupe B (Fig. 3). II s'agit d'une alternance de couches épaisses de sables et de bandes de marnes grises. Ces bandes marneuses, épaisses de 5 à $15 \mathrm{~cm}$, sont riches en microfossiles et contiennent le petit foraminifère benthique Nuttallides truempyi, caractéristique des tranches d'eau se situant entre 500 et $1000 \mathrm{~m}$ de profondeur (van Morkhoven et al., 1986). Les sables sont le plus souvent assez grossiers jaunes, même roux, contenant du gravier fin (diamètre de 4 à $5 \mathrm{~mm}$ ) surtout à la base et de lits gréseux minces. Les sables grossiers à couleurs vives proviennent probablement de l'érosion d'une nappe chevauchante qui émerge au sud de la région étudiée. Les débris organiques, tels que les huîtres et échinodermes (Flicoteaux, 1972) sont les témoins de l'érosion d'une plate-forme carbonatée proche. Le granoclassement des éléments détritiques (bases plus grossières, le reste assez homogène) et leur alternance avec des marnes d'eaux profondes indiquent un milieu turbiditique proximal.

Les Marnes de Latapy sus-jacentes affleurent sporadiquement à la suite, entre $70 \mathrm{~m}$ et $120 \mathrm{~m}$. Comme le chemin fait une grande courbe à cet endroit, elles affleurent sur une grande distance (au moins $40 \mathrm{~m}$ ), ce qui donne une impression fausse de leur épaisseur. Cependant, l'épaisseur généralement admise de 15 m (Flicoteaux, 1972 ; Sztràkos et al., 1997) pourrait être légèrement sous-estimée. Ces marnes gris clair, très riches en microfossiles semblent être déposées sous une tranche d'eau de plus de $500 \mathrm{~m}$ (présence de Bulimina trinitatensis et certaines Ammodiscus). Comparé à celui des Sables de Guillempau, le milieu de dépôt des Marnes de Latapy devient plus calme et plus éloigné des rivages.

Les Marnes de Latapy sont surmontées par le Membre de Néez. Ceci débute par le banc calcaire entre 125 et $140 \mathrm{~m}$ et s'étend jusqu'à $232 \mathrm{~m}$, au sommet du dernier banc calcaire épais de $8 \mathrm{~m}$. Ce dernier banc contient quelques niveaux marneux minces. Les deux barres calcaires détritiques, parfois 


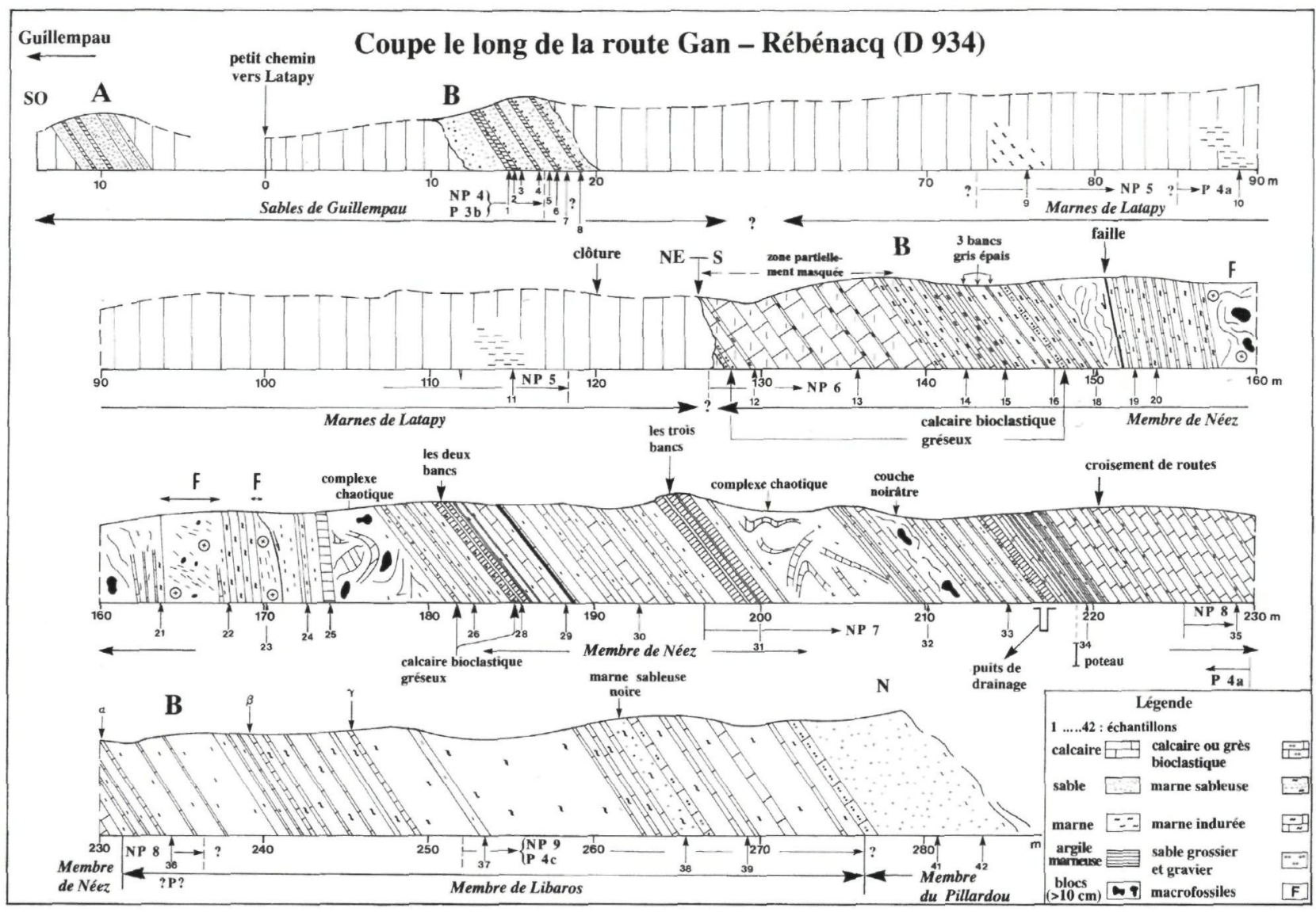

FIG. 3. - La série paléocène entre le hameau de Guillempau et le croisement avec la route Gan-Rébénacq (coupes A et B) et localisation des échantillons.

The Paleocene succession between the Guillempau hamlet and the junction with the Gan-Rébénacq road (sections A and B) with location of the samples.

micritiques, témoignent d'un milieu beaucoup plus proximal, du type infralittoral. Entre ceux-ci se trouve une alternance de marnes beiges, occasionellement noirâtres et de bancs calcaires, parfois bioclastiques. On notera la présence de grands foraminifères dans certains niveaux marneux au milieu de l'unité, apportés par des courants turbiditiques. Certaines zones sont fracturées ou présentent de structures de glissements sous-aquatiques ("slumping »). L'alternance de marnes beiges, de minces couches de calcaire et grès (10 à $15 \mathrm{~cm}$ d'épaisseur) jusqu'à $276 \mathrm{~m}$ dans la coupe $\mathrm{B}$, correspond au Membre de Libaros. Les parties inférieure et supérieure de cette unité contiennent plusieurs bandes de calcaires minces, tandis que sa partie médiane en est presque dépourvue. Le Membre de Libaros affleure également dans la coupe $\mathrm{D}$, où on observe la même subdivision. Il existe cependant des légères différences entre les deux coupes. Le banc de grès bioclas- tique à base érosive et couleur de rouille (couche $X$, voir Fig. 4), bien marqué dans la coupe $\mathrm{D}$ à une dizaine de mètres au-dessus de la base du Membre de Libaros, est à peine répérable dans la coupe $\mathrm{B}$. Ce banc, qui dans la coupe $\mathrm{D}$ atteint une épaisseur de $50 \mathrm{~cm}$ et contient des éléments remaniés grossiers à la base (2 à $3 \mathrm{~cm}$ de diamètre) et des grains de glauconie, semble se transformer latéralement, dans la coupe $B$, en banc de grès rougeâtre, épais de $15 \mathrm{~cm}$ (couche $\beta$ ). Un niveau de grès quasi identique (couche $\alpha$ ) se trouve plus bas dans la coupe $B$, à environ $1 \mathrm{~m}$ au-dessus de la base du Membre de Libaros. Une trentaine de mètres plus au nord, une marne sableuse noirâtre, légèrement gréseuse, riche en débris d'organismes, vient s'intercaler dans les marnes beiges. Nous avons reconnu ce niveau $1 \mathrm{~km}$ plus à l'ouest dans un talus de chemin près la maison Pillardou (feuille 1545 est, Pau ; $\mathrm{x}=377.725$; $\mathrm{y}=$ 3104.000), $11 \mathrm{~m}$ en-dessous de la base sableuse du 
Membre du Pillardou. Les sables sus-jacentes entre 276 et $286 \mathrm{~m}$ de la coupe B, qui contiennent des passées marneuses minces, ainsi que le grès bioclastique massive à leur base, appartiennent au Membre du Pillardou. Les marnes grisâtres, échantillonnées un peu plus au nord (éch. C1 à C3) appartiennent également à ce Membre.

Plus au nord, la série se répète à partir de la faille qui passe entre les échantillons C3 et C4 (Fig. 4). Ainsi réapparaissent les Marnes de Latapy (éch. C4), surmontées par un calcaire gréseux bioclastique rougeâtre, épais de $0.8 \mathrm{~m}$ (calcaire A : éch. C5), puis, au-dessus d'une lacune d'observation d'une centaine de mètres, le reste du Membre de Néez (éch. C6-7 et partie basale de la coupe D : éch. 1 jusqu'à 3 ) et enfin le Membre de Libaros (le reste de la coupe D). Ce calcaire gréseux A pourrait représenter la base du Membre de Néez, mais aussi bien un passage rapide dans les Marnes de Latapy, tel qu'observé dans le forage d'Artigueloutan (Sztràkos et al., 1997). Le Membre de Libaros continue jusque dans la base de la coupe E (éch. E1). Les marnes grisâtres sus-jacen- tes, affleurant juste en face du chemin du Vieux Moulin (éch. E2) appartiennent déjà à une partie élevée du Membre du Pillardou. Le reste de la coupe E, entre les échantillons E3 et E22 correspond à la partie supérieure des Marnes d'Artigueloutan. II s'agit d'une série monotone de marnes silteuses grisâtres. Les Marnes de Gan sus-jacentes, qui affleurent au gisement «Bourbon Bluff» (Fig. 2), sont nettement plus argileuses et de couleur bleuâtre. Elles peuvent être suivies plus au nord, le long des routes (p.e. au lieu-dit le Ten), jusque dans la carrière de la Tuilerie de Gan (Fig. 1). Ces dépôts deviennent plus sableux à partir du sommet de cette carrière jusqu'aux environs de la gare de Gan, un peu plus au nord. La base des Marnes de Gan affleure sur les bords du ruisseau Las Hiès (cité comme Lashies dans Gubler et Pomeyrol, 1946), 2 km à l'ouest de la maison Bourbon (coupes entre $\mathrm{x}=377.375, \mathrm{y}=3104.950$ et $\mathrm{x}=377.375, \mathrm{y}=3105.125)$. Elle est représentée par des sables fins glauconieux, dans lesquels vers le haut viennent s'intercaler des lits de marnes, pour passer enfin à des marnes bleuâtres.

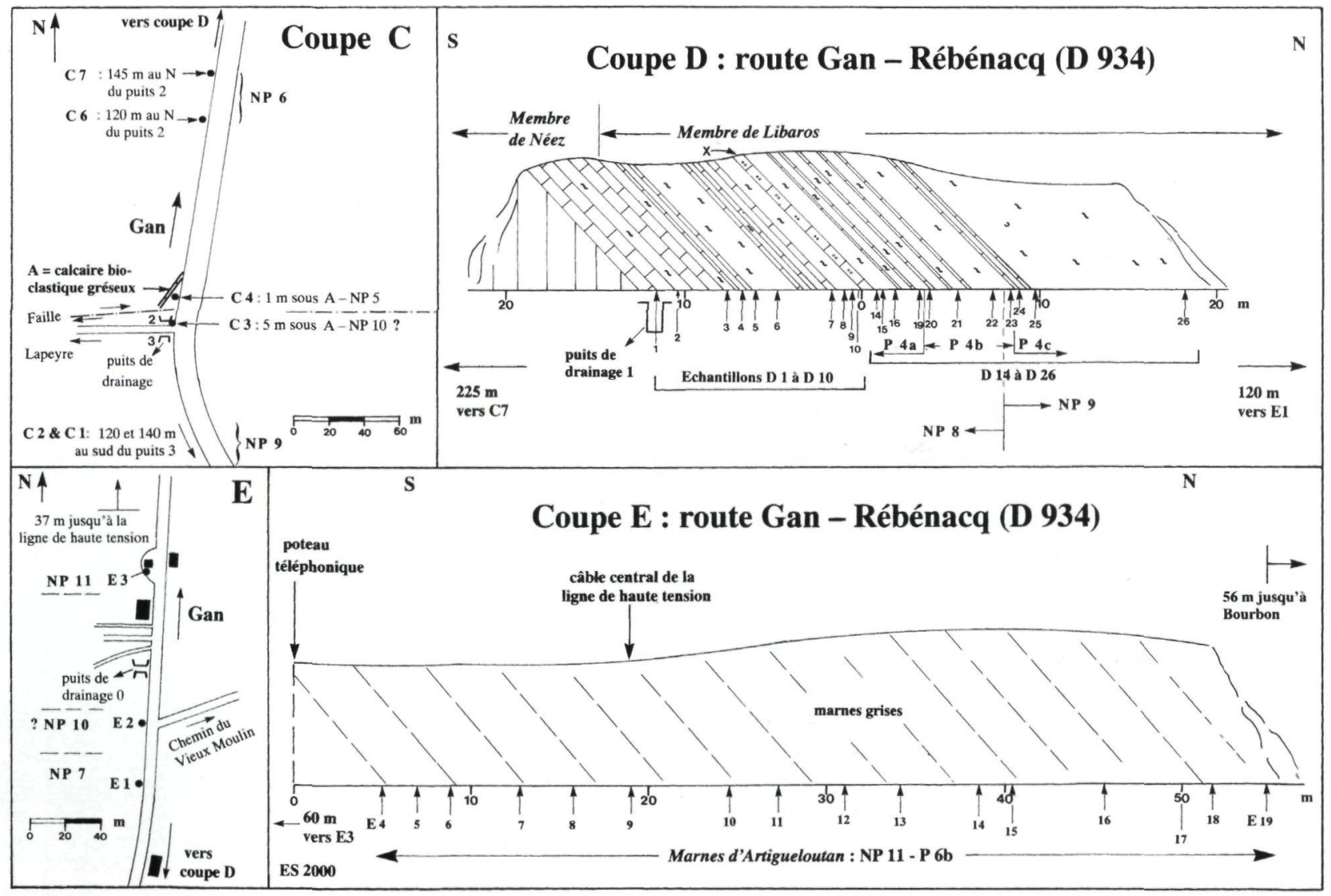

FIG. 4. - La série le long de la route Gan-Rébénacq (coupes C, D et E) et localisation des échantillons.

The succession along the Gan-Rébénacq road (sections $C, D$ and $E$ ) with location of the samples. 


\section{LES NANNOFOSSILES CALCAIRES}

\section{CADRE GÉNÉRAL}

Encouragés par les résultats positifs des premiers prélèvements (Martini, 1961 ; Hay, 1964), Hay et Mohler (1967) sont les premiers à décrire de façon détaillée les associations des nannofossiles calcaires de la coupe de la route Gan-Rébénacq. Parallèlement, Lezaud (1967) a présenté les résultats d'une série de huit échantillons de la même provenance, qu'il attribue à trois zones à nannofossiles, dont une est difficile à interpréter et les deux autres se rapportent respectivement à la NP 6 et à la NP 9. Un an plus tard il a publié les espèces nouvelles de cette étude (Lezaud, 1968). Martini (1971) a repris toutes les données concernant la coupe de la route Gan-Rébénacq dans sa monographie sur les zones à nannofossiles calcaires, en y choisissant les localités-types des zones NP 5, NP 6 et NP 7. Les nannofossiles des affleurements classiques des Marnes de Gan ont été revus par Kapellos et Schaub (1973), ceux de la tuilerie de Gan par Bigg (1982). Romein (1979) a réexaminé les échantillons 827 et 837 de Hay et Mohler et a constaté que l'échantillon supérieur se rattache plutôt à la zone NP 10 qu'à la zone NP 9 .

L'élément faible dans tous ces travaux antérieurs (y compris celui de Seyve en 1984) est la localisation imprécise des échantillons. Comme ceux-ci ne sont pas inserrés dans des levés lithologiques détaillés et que leur localisation est impossible à répérer exactement suite aux changements subséquents de la configuration des routes dans le secteur, la plupart des données micropaléontologiques anciennes ajoutent peu à la reconstitution de l'histoire géologique de la région. Le travail de Sztràkos et al. (1997) en fait l'exception, présentant pour la première fois un cadre lithostratigraphique bien défini, basé sur une combinaison de données de forages et d'affleurements bien localisés. Mais, le nombre restreint d'échantillons étudiés, qui proviennent des déblais de forage (seulement 7 sur $200 \mathrm{~m}$ de coupe dans le puits Artigueloutan 101) et le problème d'une éven-

\section{PLANCHE 1}

Agrandissements : X 115, excepté Fig. 9: X 57.

1. Chiloguembelina wilcoxensis (Cushman et Ponton, 1932); éch. C3.

2. Chiloguembelina midwayensis (Cushman, 1940); éch. D18.

3. Igorina albeari (Cushman et Bermudez, 1949) ; éch. C7.

4-5. I. tadjikistanensis (BYкоvA, 1953); éch. C7.

6. Subbotina triloculinoides (PLUMMER, 1927) ; éch. D20.

7. S. triangularis (WHITE, 1928); éch. D19.

8. S. triangularis (WHITE, 1928); éch. D10.

9. S. triangularis (WHITE, 1928) ; éch. D18.

10. S. cf. linaperta (Finlay, 1939); éch. Plbis.

11. S. patagonica (TODD et KNIKER, 1952); éch. B37.

12. S. velascoensis (CuSHMAN, 1925); éch. B15.

13. Globanomalina pseudomenardii (BoLLI, 1957) ; éch. B12.

14. G. chapmani (PARR, 1938); éch. B15.

15. G. imitata (SubBotina, 1953) ; éch. C2.

16. Acarinina coalingensis (Cushman et HanNa, 1927) ; éch. B39.

17-18. A. mckannai (WHITE, 1928) ; éch. B15.

19-20. A. subsphaerica (SubBotina, 1947) ; éch. C12.

21. A. "chascanona" (LOEBLICH et TAPPAN, 1957); éch. B12.

22. A. nitida (Martin, 1943); éch. B15.

23. A. soldadoensis soldadoensis (BRöNNIMANN, 1952) ; éch. El.

24. A. soldadoensis soldadoensis (BRÖNNIMANN, 1952) ; éch. B39. 


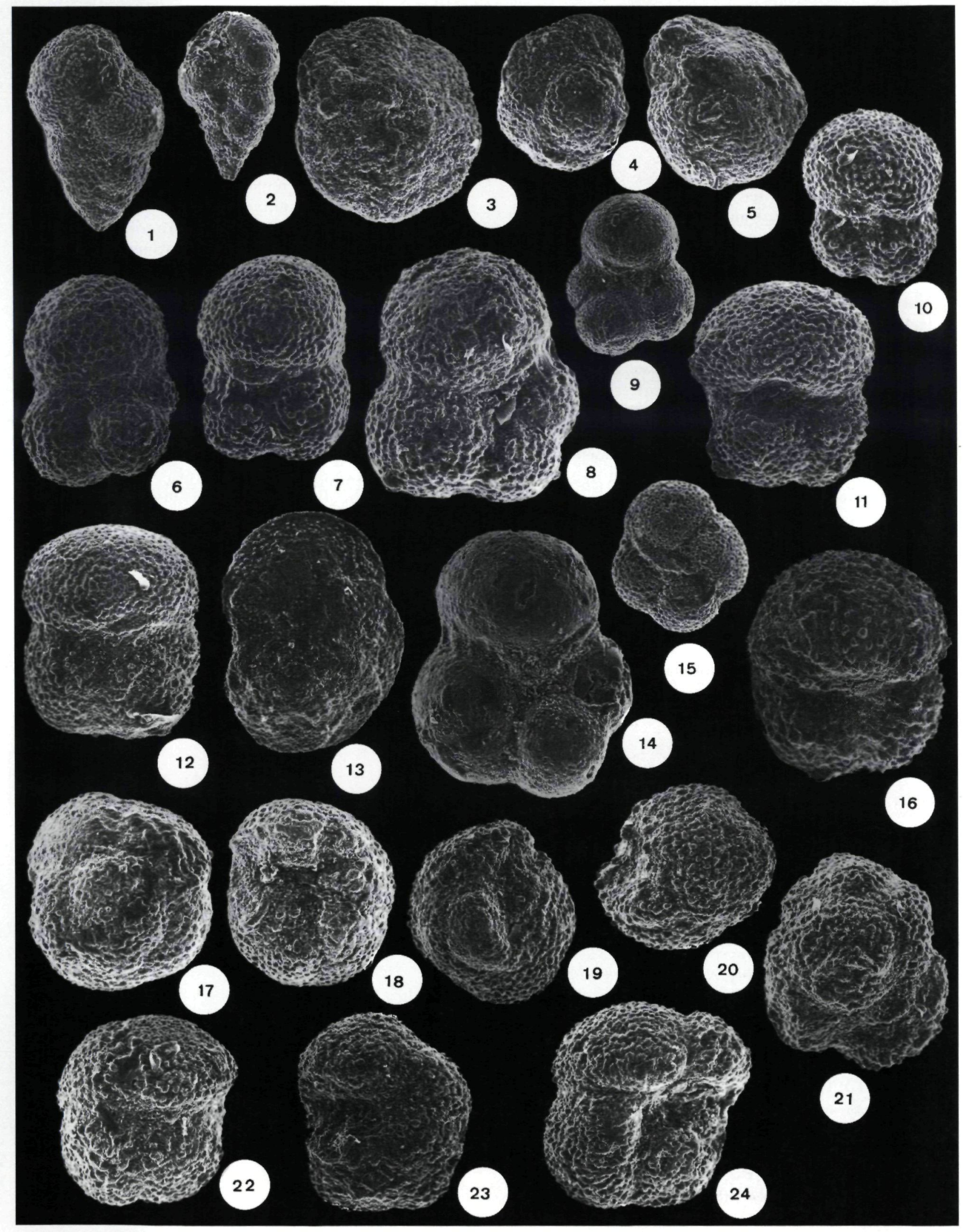


tuelle contamination de ce matériel restent sujette à caution, vu certaines discordances entre ce travail et celui que nous présentons ici.

\section{TAXINOMIE ET BIOZONATION ADOPTÉES}

La taxinomie adoptée est en principe celle de Perch-Nielsen (1985), tenant compte des modifications apportées par Varol (1992) (Sullivania) et Aubry (1999) (Blackites). La zonation que nous utilisons ici est celle de Martini (1971) et en partie celle de Varol (1989), définie dans le Paléocène du Bassin de la Mer du Nord, mais qui pour certains intervalles semble aussi bien applicable dans des régions plus méridionales (par exemple la Tunisie, Steurbaut et $a l ., 2000)$. L'interprétation biostratigraphique du Membre du Pillardou est basée sur les séquences d'événements reconnues en région méditerranéenne (Monechi et al., 2000a et b), essentiellement en Egypte (von Salis et al., 1998 ; Aubry et al., 1999). Ces zonations sont calibrées à celle de Martini (1971 : les zones standards NP, prouvées mondialement utilisables), étant essentiellement basée sur les mêmes marqueurs. Cependant, les zonations de Varol, von Salis et al. et Aubry et al. sont beaucoup plus détaillées, s'appuyant sur un plus grand nombre de marqueurs. La répartition des espèces à intérêt stratigraphique, ainsi que la biozonation de l'intervalle étudié, sont illustrées dans la Figure 5. La liste alphabétique des espèces discutées dans le texte est donnée en annexe.

\section{INTERPRÉTATION DES ASSOCIATIONS}

Les minces bandes d'argile dans la partie inférieure des Sables de Guillempau sont riches en nannofossiles (éch. B1 à B4; B5 à B8 sont stériles), malgré l'absence d'un certain nombre de petites formes dans quelques échantillons à cause d'une décalcification prononcée (en moyenne 25 espèces autochtones par échantillon et entre 15 et 45 spécimens $/ \mathrm{mm}^{2}$; spécimens calculés suivant les méthodes de Backman et Shackleton, 1983). Parmi les espèces les plus diagnostiques on notera Ellipsolithus distichus, E. macellus, Sphenolithus primus, Fasciculithus janii, F. billii, F. pileatus, F. ulii, Bomolithus elegans, Biantholithus sparsus, Neochiastozygus digitosus et Toweius tovae. La présence de $F$. janii, $F$. ulii et $F$. billii, associée à l'absence de Fasciculithus tympaniformis indique la sous-zone à Fasciculithus janii ou NTp8C de Varol (1989), qui correspond à la partie sommitale de la zone NP 4 de Martini (1971).

Les Marnes de Latapy sont plus riches en nannofossiles (éch. B9 à B11 : en moyenne 70 specimens $/ \mathrm{mm}^{2}$ ), dont la préservation est nettement meilleure. Les associations sont dominées par les Prinsiaceae. Par rapport à celles des Sables de Guillempau, elles sont nettement moins riches en Fasciculithus. Elles sont caractérisées par l'apparition de Prinsius bisulcus, T. pertusus, Scapholithus apertus et Fasciculithus tympaniformis. La présence de cette dernière espèce, associée à l'absence de Heliolithus kleinpellii indique la zone NP 5. Les premiers exemplaires de Toweius eminens apparaissent dans

\section{PLANCHE 2}

Agrandissements : Fig. 1-4, 6-7, 9-12, 15 : X 115 ; Fig. 5, 8, 13, 14 : X 57.

1. Acarinina whitei (WEISS, 1955); éch. D19.

2. Acarinina whitei (WEISS, 1955); éch. D8.

3. A. wilcoxensis (CUSHMAN et PONTON, 1932) ; éch. C1.

4. A. wilcoxensis (CUSHMAN et PONTON, 1932) ; éch. C3.

5. Morozovella aequa (CUSHMAN et RENZ, 1942); éch. B15.

6-7. M. aequa (CUSHMAN et RENZ, 1942); éch. C2.

8. M. acuta (TOULMIN, 1941); éch. C3.

9-10. M. apanthesma (LOEBLICH et RENZ, 1957) ; éch. D6.

11-12. M. apanthesma (LOEBLICH et RENZ, 1957) ; éch. C12.

13. M. occlusa (LOEBLICH et TAPPAN, 1957) ; éch. D8.

14. M. velascoensis (CUSHMAN, 1925) ; éch. B33.

15. M. velaseoensis (CUSHMAN, 1925) ; éch. D8. 


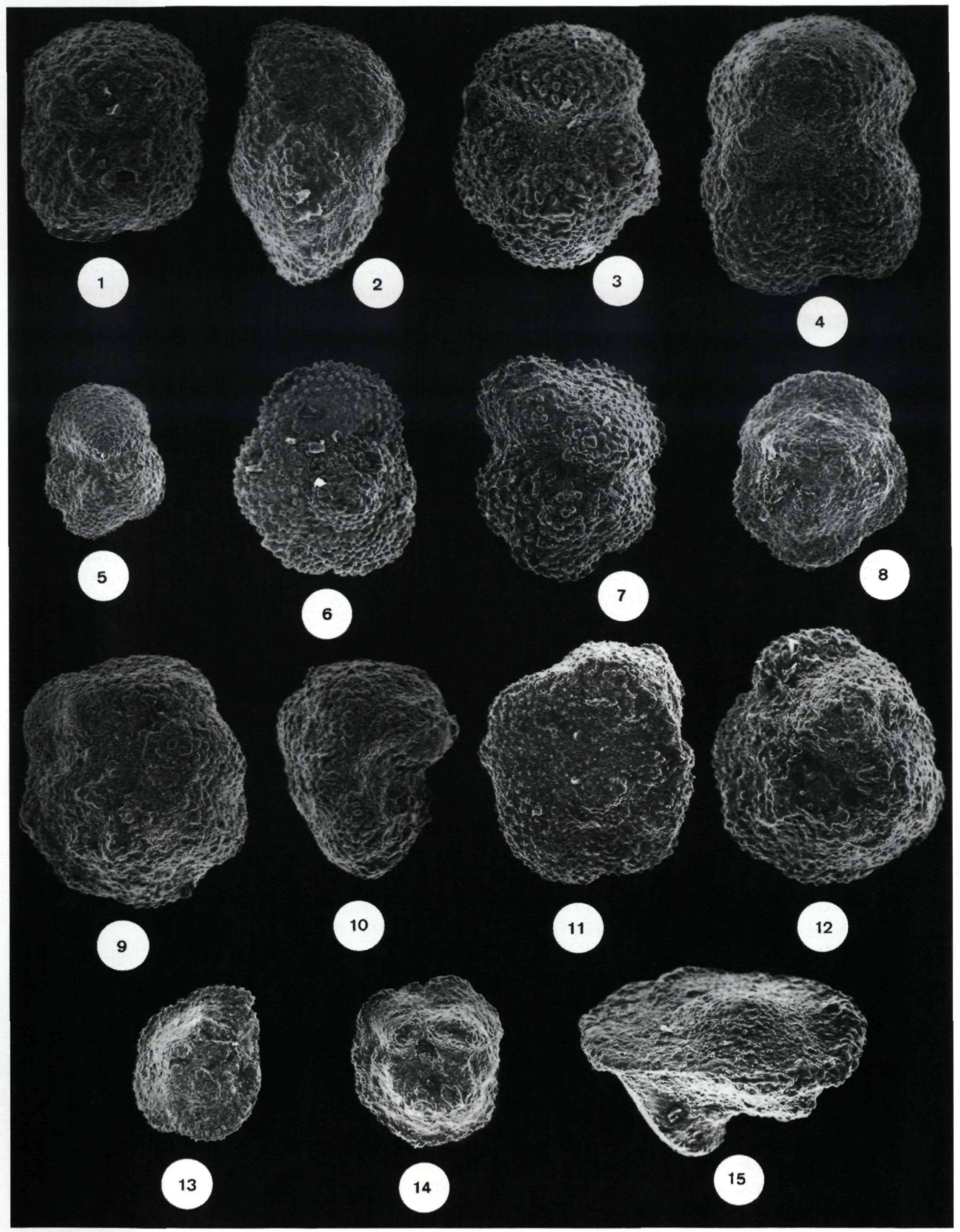


l'échantillon B10. Le contact avec le Membre de Néez sus-jacent n'a pas été observé dans la coupe B. La base érosive du grès grossier rougâtre affleurant dans la coupe C (éch. C5) pourrait représenter ce contact. Si c'est vrai, la partie sommitale des Marnes de Latapy, représentée par l'échantillon $\mathrm{C} 4,1 \mathrm{~m}$ en dessous du grès, appartient encore à la zone NP 5 . Si ce banc de grès fait encore partie du Membre de Latapy, il n'est pas exclu qu'une partie de ces marnes soit attribuable à la zone NP 6.

Le premier échantillon du Membre du Néez (éch. B12) est marqué par la présence de Heliolithus kleinpellii et l'absence de Discoaster mohleri, permettant une attribution à la zone NP 6 . Les associations de la partie inférieure de ce membre (jusqu'à l'échantillon B 19) sont assez mal conservées. Fasciculithus janii est encore présent dans la barre calcaire inférieure. Cette barre contient d'assez fréquents Braarudosphaera, quasi-absents dans les couches qui l'encadrent, indiquant une origine plus proximale. Les premiers Discoasteroides bramlettei, Neochiastozygus distentus et Zygodiscus herlynii apparaissent juste au-dessus du niveau repère "les deux bancs» (éch. B29). Les premiers Discoaster mohleri, marquant par définition la base de la zone NP 7 de Martini, ont été signalés dans les trois bancs, niveau répère au milieu du Membre du Néez (éch. B31), environ $15 \mathrm{~m}$ plus haut. Ce niveau contient encore beaucoup de $\boldsymbol{H}$. kleinpellii. Cette espèce est présente pour la dernière fois dans l'échantillon suivant (B32). Heliolithus knoxii est sporadiquement présent dans B33 et B34. Discoaster mohleri devient fréquent au moment où Heliolithus riedelii, marqueur de la base de la zone NP 8 , fait son apparition. Ceci se situe au sommet de la barre supérieure du Membre de Néez (éch. B35 : 15 exemplaires de $D$. mohleri sur 10 champs de vue à agrandissement 1000x ; en dessous de la barre moins d'un exemplaire sur 10 champs de vue). H. riedelii (représentée souvent par des exemplaires à bords légèrement corrodés) persiste jusque dans la base du Membre de Libaros (éch. B36). Les premiers Discoaster multiradiatus, aux caractères primitifs (la moyenne du nombre de rayons est 31 ; selon Romein $(1979$, p. 84) un chiffre aussi élevé ne se retrouve que dans les populations les plus anciennes), apparaissent au milieu du Membre de Libaros (éch. B37). Cette espèce, marqueur de la base de la zone NP 9 , s'y trouve en compagnie des derniers Ericsonia universa. La marne sableuse noirâtre dans la partie supérieure du Membre de Libaros (éch. B38) est caractérisée par l'apparition de Discoaster falcatus, D. nobilis, D. splendidus et Campylosphaera eodela, et

\title{
PLANCHE 3
}

\author{
Les lames et les négatifs des photos des planches 3 et 4 sont conservés dans les archives de l'Institut Royal des Sciences naturelles de Belgique, \\ rue Vautier 29, B-1000 Bruxelles, Belgique. Les numéros donnés aux spécimens figurés y refèrent. La barre sur chaque photo représente 5 \\ $\mu \mathrm{m}$ (à l'exception de celle de la Pl. 2, Fig. 1 qui fait $1 \mu \mathrm{m}$ ). Abréviations utilisées : MEB = microscope électronique à balayage ; .p. $=$ nicols \\ parallèles, fond clair ; n.c. $=$ nicols croisés, fond noir. \\ 1. Heliolithus kleinpellii Sullivan, 1964 ; a = n.c., b = n.p. ; éch. B26 ; NP 6 (IRScNB b4009). \\ 2. Discoaster multiradiatus BRAMLETTE et RIEDEL, 1954 ; MEB ; éch. B39 ; NP 9 (IRSeNB b4010). \\ 3. Discoaster multiradiatus BRAMLETTE et RIEDEL, 1954 ; MEB ; éch. B39; NP 9 (IRScNB b4011). \\ 4. Discoaster mahmoudii Perch-Nielsen, 1981 ; n.p. ; éch. E2, ?NP 10 (IRSeNB b4012). \\ 5. Discoaster mahmoudii Perch-Nielsen, 1981 ; n.p. ; éch. E2, ?NP 10 (IRSeNB b4013). \\ 6. Toweius eminens (Bramlette et Sullivan, 1961) Perch-Nielsen, 1971 ; MEB ; éch B39 ; NP 9 (IRScNB b4014). \\ 7. Discoaster lodoensis BraMLETTE et RIEDEL, 1954 ; n.p. ; Gan, tuilerie B ; NP 13 ; (IRSeNB b4015). \\ 8. Discoaster ef. nobilis MARTinI, 1961 ; MEB ; éch. B39; NP 9 (IRScNB b4016). \\ 9. Ericsonia universa (WIND et WISE, 1977) RoMeIN, $1979 ; \mathrm{a}=$ n.p. ; b = n.c. ; éch. B37 ; NP 9 (IRScNB b4017). \\ 10. Fasciculithus janii Perch-NieLSEN, 1971 ; a = n.p. ; b = n.c. ; éch. B1; NP 4 (IRSeNB b4018). \\ 11. Fasciculithus janii Perch-Nielsen, 1971 ; MEB ; éch. B2 ; NP 4 (IRScNB b4019). \\ 12. Discoaster multiradiatus BRAMLETTE et RIEDEL, 1954 ; n.c. ; éch. B39; NP 9 (IRSeNB b4020). \\ 13. Micrantholithus sp. ; n.c. ; éch. E2 ; ? NP 10 (IRScNB b4021). \\ 14. Heliolithus riedelii Bramlette et Sullivan, 1961 ; n.c. ; éch. D2 ; NP 8 (IRScNB b4022). \\ 15. Heliolithus riedelii BraMlette et Sullivan, 1961 ; n.c. ; éch. B35 ; NP 8 (IRScNB b4023). \\ 16. Semihololithus kerabyi Perch-Nielsen, 1971 ; n.c. ; éch. C1 ; NP 9 (IRScNB b4024). \\ 17. Zygodiscus herlynii Sullivan, 1964 ; n.c. ; éch. B38; NP 9 (IRSeNB b4025). \\ 18. Blackites solus (Perch-NIElsen, 1971) AUbry, 1999 ; n.c. ; éch. C3 ; ?NP 10 (IRScNB b4026). \\ 19. Discoaster splendidus MARTINI, 1960 ; n.p. ; éch. B39 ; NP 9 (IRSeNB b4027).
}



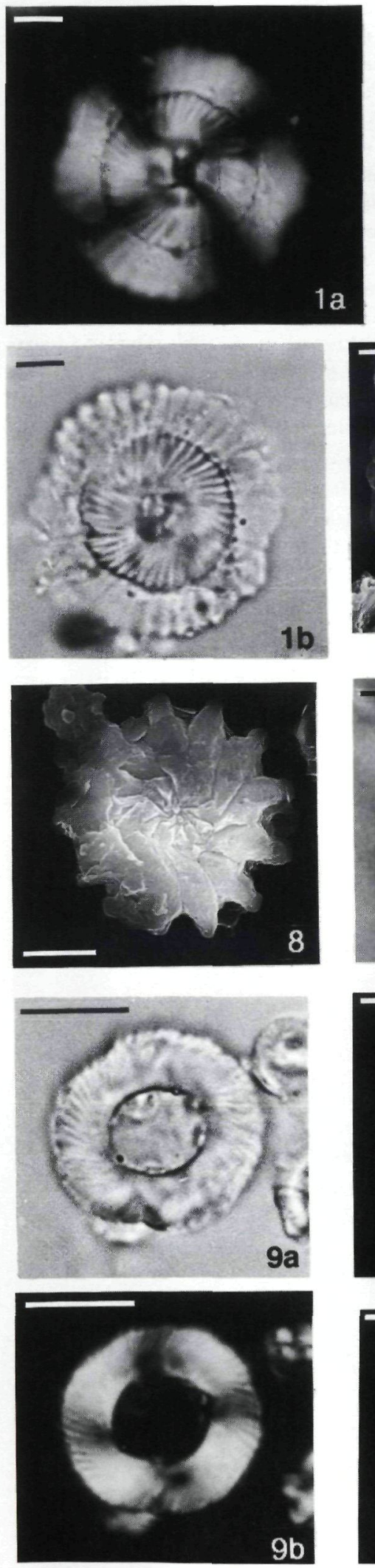
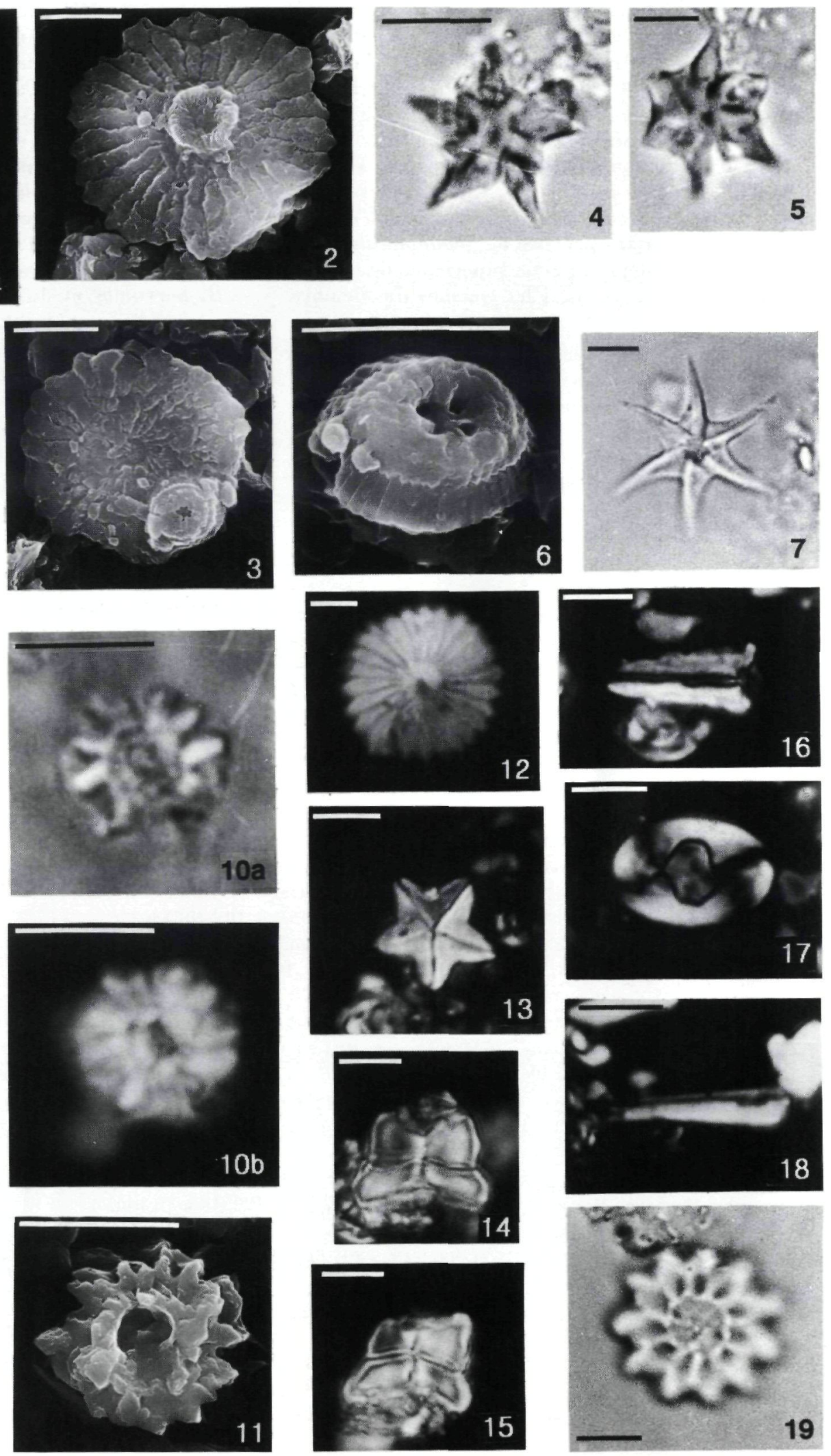
par la présence d'assez nombreux Zygrhablithus bijugatus et Braarudosphaera bigelowii. Ces dernières espèces indiquent des eaux plus proches du rivage. L'association de cette couche noirâtre est attribuable à la partie moyenne de la sous-zone NP9a de Aubry et al., 2000 (= sous-zone NP9b de von Salis et al., 1998). La partie sommitale du Membre de Libaros (éch. B39) contient des Discoaster lenticularis, Fasciculithus alanii, Heliolithus megastypus et Toweius serotinus. Des formes proches de Semihololithus kerabyi annoncent déjà la partie supérieure de la zone NP 9a, bien exprimée dans les couches du Membre du Pillardou sus-jacent.

La partie basale grossière du Membre du Pillardou est dépourvue de nannofossiles calcaires. Une zone marneuse située plus haut (éch. C1 et C2) est marquée par plusieurs apparitions, parmi lesquelles $S$. biskayae (fréquent) et $S$. kerabyi (fréquent), par la présence de Pontosphaera spp. et la fréquence de Fasciculithus, aussi bien en espèces qu'en individus (e.a. les premiers $F$. schaubii et $F$. sidereus). Les premiers Rhomboaster cuspis apparaissent dans l'échantillon C2. La présence de ces formes, associée à l'absence de Discoaster anartios, D. araneus et Rhomboaster calcitrapa suggère la partie supérieure de la sous-zone NP9a. Les marnes affleurant $100 \mathrm{~m}$ plus au nord, dans le versant de la route vers Lapeyre (éch. C3), s'en distinguent par la dominance des formes plus côtières, comme Zygrhablithus bijugatus, Braarudosphaera bigelowii, Trochoaster operosum et Micrantholithus spp., par la forte diminution en nombres d'espèces et d'individus du genre Fasciculithus, et par l'apparition de Blackites solus, $B$. herculeus et de rares Discoaster diastypus. Les taxons Neocrepidolithus spp., S. biskayae, S. kerabyi et $S$. apertus ne sont plus présents (à l'exception de quelques rares specimens remaniés). Cette association pourrait indiquer la base de la zone NP 10.

Comme déjà discuté plus haut, la série se répète par la suite d'une faille, située à quelques mètres au nord de l'échantillon C3. Les Marnes de Latapy, juste en dessous du calcaire gréseux A (éch. C4) se rattachent à la zone NP 5. Les échantillons C6 et C7, $100 \mathrm{~m}$ plus au nord, sont caractérisés par la présence

\section{PLANCHE 4}

1. Campylosphaera eodela Bukry et Percival, 1971 ; MEB ; éch. B39 ; NP 9 (IRScNB b4028).

2. Campylosphaera eodela BUKRY et Percival, 1971 ; n.c.; éch. B39 ; NP 9 (IRSeNB b4029).

3. Tribrachiatus orthostylus SHAMraI, 1963 ; n.p. ; éch. E3 ; NP 11 (IRScNB b4030).

4. Tribrachiatus orthostylus SHAMraI, 1963 ; n.p. ; éch. E3 ; NP 11 (IRSeNB b4031).

5. Rhomboaster cuspis BRamlette et Sullivan, 1961 ; n.p. ; éch. C3 ; ? NP 10 (IRSeNB b4032).

6. Rhomboaster cuspis Branlette et SUllivan, 1961 ; n.p. ; éch. E3 ; NP 11 (IRSeNB b4033).

7. Toweius eminens (Bramlette et Sullivan, 1961) Perch-Nielsen, 1971 ; n.c. ; éch B38 ; NP 9 (IRScNB b4034).

8. Fasciculithus janii Perch-NIELSEN, 1971 ; n.c. ; éch. B12, NP 6 (IRSeNB b4035).

9. Rhomboaster sp. ; n.p. ; éch. E2 ; ? NP 10 (IRSeNB b4036).

10. Fasciculithus pileatus BUKRY, 1973 ; n.c. ; éch. B1 ; NP 4 (IRSeNB b4037).

11. Fasciculithus ulii PERCh-NIELSEN, 1971 ; n.c. ; éch. B1 ; NP 4 (IRSeNB b4038).

12. Fasciculithus ulii Perch-NIELSEN, 1971 ; n.c. ; éch. B4 ; NP 4 (IRScNB b4039).

13. Fasciculithus ulii Perch-Nielsen, 1971 ; n.c. ; éch. B2 ; NP 4 (IRSeNB b4040).

14. Prinsius bisulcus (Stradner, 1961) Hay et Mohler, 1967 ; n.c. ; éch. B10 ; NP 5 (IRScNB b4041).

15. Rhomboaster cuspis Bramlette \& Sullivan, 1961 ; n.p. ; éch. C3 ; ? NP 10 (IRScNB b4042).

16. Discoasteroides bramlettei BUKRY et PERCIVAL, 1971 ; n.p. ; éch. B30 ; NP 6 (IRSeNB b4043).

17. Discoasteroides bramlettei Bukry et Percival, 1971 ; n.c. ; éch. B30 ; NP 6 (IRScNB b4044).

18. Discoaster mohleri Bukry et Percival, 1971 ; n.p. ; éch. B31; NP 7 (IRScNB b4045).

19. Fasciculithus schaubii HAY et MOHLER, 1967 ; n.p. ;éch. C2 ; NP 9 (IRScNB b4046).

20. Fasciculithus schaubii HaY et MoHLeR, 1967 ; n.c. ; éch. C1 ; NP 9 (IRScNB b4047).

21. Neococcolithes protenus (Bramlette et Sullivan, 1961) BLACK, 1967 ; n.p. ; éch. C2 ; NP 9 (IRScNB b4048).

22. Discoaster diastypus Bramlette et Sullivan, 1961 ; n.c. ; éch. E2 ; ?NP 10 (IRScNB b4049).

23. Heliolithus knoxii Steurbaut, 1998 ; a = n.p. ; b = n.c., éch. B33 ; NP 7 (IRScNB b4050).

24. Fasciculithus tympaniformis HAY et MOHLER in HAY et al. 1967 ; n.c. ; éch. B10 ; NP5 (IRScNB b4051).

25. Scapholithus apertus HAY et MOHLER, 1967 ; n.c. ; éch. B9 ; NP5 (IRScNB b4052).

26. Semihololithus biskayae Perch-NIELSEN, 1971 ; n.e. ;éch. C1 ; NP 9 (IRScNB b4053).

27. Bomolithus elegans Roth, 1973 ; n.c. ; éch. B1 ; NP 4 (IRScNB b4054). 

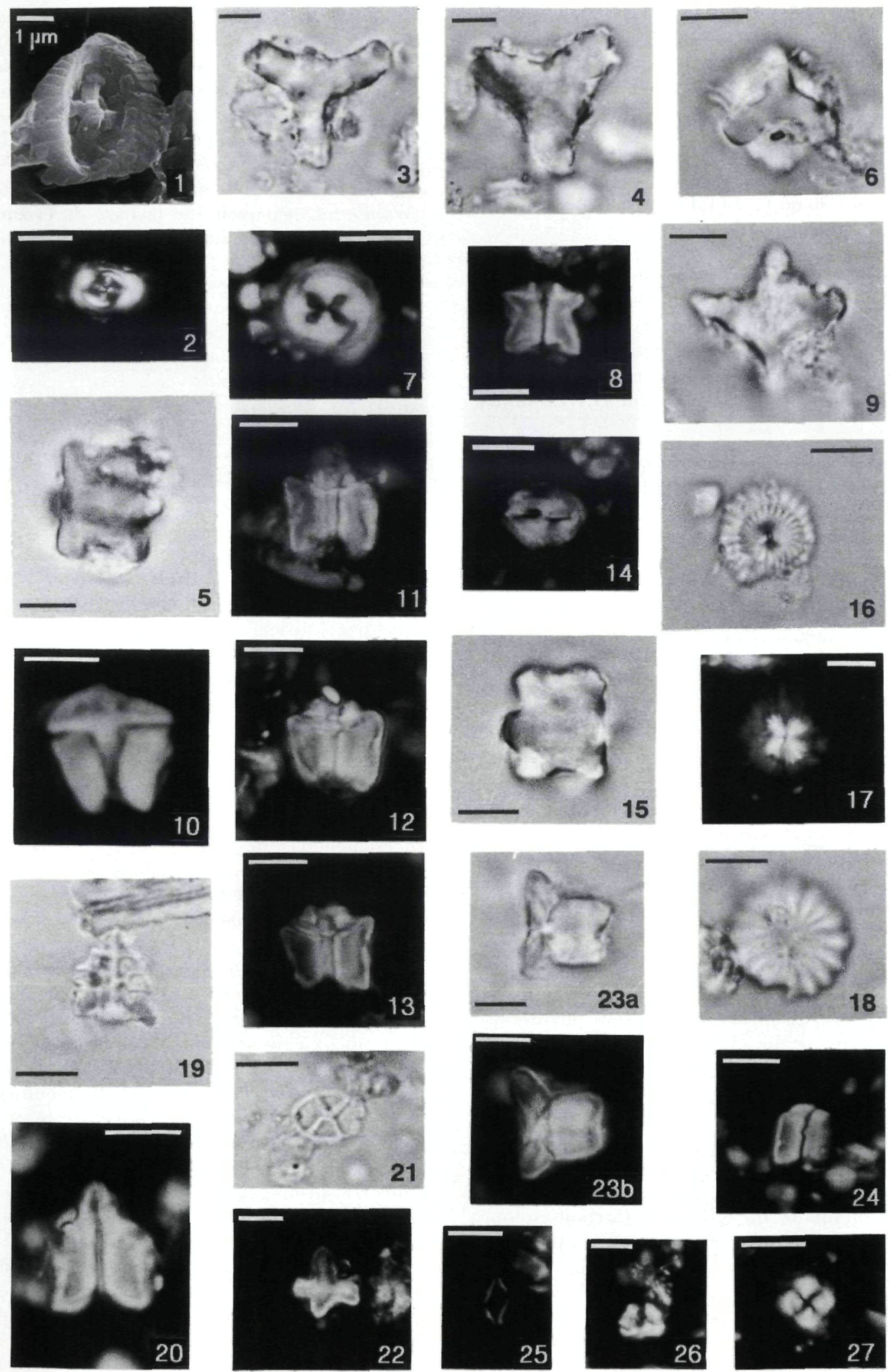

REVUE DE MICROPALÉONTOLOGIE, VOL. 45, No 3 
de H. kleinpellii et D. bramlettei, ce qui permet leur attribution à la partie sommitale de la zone NP 6 , et par corrélation avec la coupe $B$, à leur inclusion dans la partie moyenne du Membre de Néez (quelque part entre les « deux bancs » et les »trois bancs»).

L'échantillonnage plus serré de la coupe D permet de préciser la position des événements décrits plus haut au sein du Membre de Libaros. L'espèce Heliolithus riedelii, marqueur de la base de NP 8, est présente dans la barre calcaire supérieure du Membre du Néez (éch. D2) et dans la première masse marneuse du Membre de Libaros (éch. D5 et D6). Ericsonia universa se rencontre à partir de l'échantillon D19, $3 \mathrm{~m}$ au-dessus du grès rougâtre $X$, jusque dans les échantillons D23 et D24, où il coexiste avec les premiers $D$. multiradiatus et les derniers Heliolithus knoxii.

Environ $120 \mathrm{~m}$ plus au nord de la coupe D affleurent des marnes (éch. El), qui par la présence de $D$. mohleri, $D$. bramlettei et $H$. knoxii, associée à l'absence de $H$. kleinpellii et $H$. riedelii, appartiennent à la partie sommitale de NP 7, et par conséquent à la partie supérieure du Membre de Néez (la portion juste en-dessous de la barre supérieure). Les deux zones d'affleurements $\mathrm{D}$ et $\mathrm{E}$ sont séparées par au moins une faille, les marnes de l'échantillon El étant nettement plus anciennes que celles de la coupe $\mathrm{D}$. L'échantillon E2, $40 \mathrm{~m}$ plus au nord, se rattache déjà à la partie supérieure du Membre du Pillardou. L'association est marquée par la fréquence de Braarudosphaera, Micrantholithus spp. et Z. bijugatus, et par la présence de Discoaster diastypus, D. mahmoudii, B. solus et $R$. cuspis. Elle ressemble de très près à celle de l'échantillon C3. Une telle association pourrait indiquer la base de la zone NP 10 (voir plus bas). Notons le nombre considérable de formes remaniées dans cette association (par exemple H. kleinpellii).

Par rapport à ce qui s'observe dans les coupes de transition paléocène-éocène en région méditerranéenne (Espagne, Italie, Egypte), les marnes de la partie inférieure du Membre du Pillardou (éch. C1 et C2) se situeraient dans la partie supérieure de la sous-zone NP 9a de Aubry et al. (2000), nettement en-dessous du niveau de l'extinction des foraminifères benthiques (le BFEE ou le " benthic foraminifera extinction event ") et de l'excursion la plus négative du $\delta^{13} \mathrm{C}$ (le minimum du CIE ou «Carbon isotope event »). Ces conclusions sont basées sur la présence des taxons S. apertus, Neocrepidolithus spp., Heliolithus megastypus, Fasciculitus alanii et $F$. schaubii dans C1 et C2. Ceux-ci disparaissent juste avant le BFEE (von Salis et al., 1998 ; Monechi et al., 2000a et b ; Aubry et al., 1999 et 2000). La présence de $D$. diastypus, D. mahmoudii et la forte diminution en Fasciculithus, aussi bien en espèces qu'en individus, suggèrent que la partie supérieure du Membre du Pillardou, représentée par les échantillons E2 et C3 se serait déposée postérieurement à l'extinction des foraminifères benthiques (le BFEE) et le CIE. Ces espèces suggèrent la présence de la zone NP 10 , mais, comme nous n'avons pas retrouvé Tribrachiatus bramlettei, marqueur de la base de la zone NP 10 , cette attribution zonale reste sujet à caution.

Les marnes grisâtres, s'étendant à partir de l'échantillon E3 jusqu'au sommet de la coupe E, représentent le Membre d'Artigueloutan. La partie inférieure de ce membre (éch. E3 à E12) contient des associations mal conservées, avec Tribrachiatus orthostylus, Discoaster barbadiensis, D. diastypus, D. multiradiatus et $B$. solus. La présence de T. orthostylus, en absence de $T$. contortus, permet leur assimilation à la base de la zone NP 11. La partie supérieure du Membre d'Artigueloutan, représentée par l'échantillon E22 pris juste au sud de la maison Bourbon, est plus riche en nannofossiles. L'association comporte T. orthostylus, E. macellus, Blackites truncatus et $Z$. bijugatus nolfii. Cet ensemble, marqué par l'absence de Discoaster lodoensis, suggère un rattachement à la partie supérieure de la NP 11, plus précisement la sous-zone III al de Steurbaut (1991).

Un peu plus au nord, derrière la maison Bourbon affleurent des marnes bleuâtres, appartenant aux Marnes de Gan. La base de ces marnes, uniquement observée dans la coupe de la rivière Las Hiès, est représentée par des sables fins. Ceux-ci contiennent des associations pauvres, dominées par des formes remaniées. La présence de D. lodoensis, Blackites creber, Helicosphaera seminulum et T. orthostylus indique la base de la partie supérieure de la zone NP 12 (base de la sous-zone VII de Steurbaut, 1991). Les niveaux marneux qui les surmontent sont nettement plus riches en nannofossiles. Ces associations sont mieux conservées, mais sont aussi plus difficiles à interpréter vue l'abondance de formes remaniées. Les couches derrière la maison Bourbon sont caractérisées par la fréquence relative de $B$. creber et $D$. lodoensis. Ceci, associé à la présence de Reticulofenestra sp., H. seminulum, T. orthostylus et Lophodolithus reniformis permet un rattachement à la base de la partie supérieure de la zone NP 12, précisement à la sous-zone VII de Steurbaut (Steurbaut, 1991 ; Schmitz et al., 1996). Le sommet de la coupe de Bourbon est marqué par les premiers exemplaires de Toweius gammation. Une association presque identique a été identifiée au lieu-dit le Ten ( $\mathrm{x}=377.900 ; \mathrm{y}=$ $3166.075)$, indiquant que ce niveau se situe dans la 
même sous-zone. L'apparition de Pentaster lisbonensis suggère quand même un âge un peu plus jeune. Les associations des couches de la carrière de la tuilerie s'en distiguent par la fréquence des Micrantholithus, avec en outre l'apparition de M. inaequalis. L'abondance de ce groupe indique des environnements plus proches du rivage. $T$. orthostylus, qui par définition s'étend jusqu'à la base de la zone NP 13 semble disparaître au sein de ces couches. Certains niveaux de la carrière ainsi que le gisement de Bosdarros $(x=380.725 ; y=3106.125)$ contiennent de rares Coccolithus crassus et Discoaster cruciformis, ce que confirme leur attribution à la zone NP 13 .

\section{COMPARAISON AVEG LES DONNÉES ANCIENNES}

Les résultats de nos recherches diffèrent en certains points de ceux présentés par Sztràkos et al. (1997), essentiellement basés sur des données anciennes et sur quelques analyses nouvelles de certains niveaux dans le forage d'Artigueloutan 101. Ils permettent de modifier les âges des différents membres. Ainsi, la base des Marnes de Latapy, considérée attribuable à la zone NP 6 par Sztràkos et al. (1997, p. 39), s'est averée un peu plus ancienne dans la présente étude (NP 5). Puis, la base du Membre de Néez se situe dans la zone NP 6 dans sa localité-type et non entre les zones NP 6 et NP 7, comme les anciennes datations l'ont laissé supposer. Cette limite est probablement une surface hétérochrone, si on tient compte des résultats du puits Artigueloutan 101 où elle se trouve entre les zones NP 6/NP 7 (Sztràkos et $a l .$, 1997). De plus, une partie du Membre de Libaros occupe la base de la zone NP 9. Le Membre du Pillardou débute plus haut dans cette zone. La limite de ces unités a été placée entre les zones NP 8 et NP 9 précédemment (Sztràkos et al., 1997).

\section{LES FORAMINIFÈRES}

\section{CADRE GÉNÉRAL}

Les foraminifères planctoniques de la coupe de Gan-Rébénacq ont été étudiés par Flicoteaux (1967), Plaziat et al. (1975) et Seyve (1984). Peybernès et al. (1996) ont publié des données sur les affleurements paléocènes et éocènes de Lasseube, dans la proximité de cette coupe. Gubler et Pomeyrol (1946) ont donné la liste des foraminifères des Marnes de Gan des localités-types, à partir des déterminations de Texier. Sztràkos (2000) a étudié des foraminifères des Marnes d'Artigueloutan et Marnes de Gan, en partie à l'aide des échantillons qui sont décrits ici. Les numéros S11 à S27 chez Sztràkos (2000) correspondent aux échantillons E3 à E19 de ce travail, le S28 au E21 et le B2 au E22. Nous ne souhaitons pas revenir sur la partie éocène de la coupe. Ce sont plutôt les échantillons paléocènes qui seront traités ici avec plus de détail.

\section{BIOZONATION ADOPTÉE}

Pour l'étude biostratigraphique, nous suivons la conception de Berggren et al. (1995) concernant la définition des biozones et celle de Berggren et Norris (1997) et Olsson et al. (1999) pour la taxonomie des genres et des espèces. Berggren et Norris et Olsson et al. considèrent que Acarinina subsphaerica et $A$. chascanona sont synonymes. Nous regroupons sous le nom $A$. "chascanona " des formes de petite taille possédant un trochospire élevé que l'on observe fréquemment au Thanétien et à l'Yprésien inférieur. Leur attribution spécifique est encore incertaine et demande des investigations supplémentaires. En Aquitaine, ce groupe d'espèces monte jusqu'à la partie supérieure de la zone $\mathrm{P} 6$, correspondant à la zone à nannofossiles NP 11 (Sztràkos, 1996 ; Sztràkos et al., 1998). La dernière présence d'Acarinina subsphaerica indique le sommet de la sous-zone P 4a, défini par Berggren et al. (1995). Ceci se situe dans la zone à nannofossiles NP 8 selon ces mêmes auteurs. Sa répartition stratigraphique se limite à la zone $P$ 4a dans les régions près de l'équateur, mais sa disparition se situe au sommet de la zone $\mathrm{P} 5$ dans l'hémisphère sud tout-à-fait méridionale (Olsson et $a l ., 1999)$. Le même phénomène s'observe dans l'hémisphère nord, dans différentes coupes européennes. Ainsi, Molina et al. (1999) ont constaté que cette espèce peut se trouver dans une position stratigraphique plus élevée, à la base de la zone NP 9, comme dans les Cordillères bétiques et au sud des Pyrénées en Espagne ainsi qu'à Possagno, en Italie du Nord. Par conséquent, la distinction des sous-zones $\mathrm{P}$ 4a et $P$ 4b est impossible dans ces régions. En révisant le Paléocène de la coupe de Gan-Rébénacq au sud de Pau, nous avons constaté que l'intervalle entre la dernière présence de cette espèce et la première apparition d'A. soldadoensis, indicatrice de la base de la sous-zone $\mathbf{P} 4 \mathrm{c}$, est réduit à $4 \mathrm{~m}$ dans la zone $\mathrm{P} 4$ dont l'épaisseur dépasse $170 \mathrm{~m}$.

\section{INTERPRÉTATION DES ASSOCIATIONS}

La liste des foraminifères planctoniques de l'ensemble de la coupe de Gan-Rébénacq est détaillée 
dans le Tableau 1. Les foraminifères planctoniques remaniés du Crétacé apparaissent sporadiquement dans le Paléocène : échantillons B25, C11. Les remaniements du Crétacé et du Paléocène (zones $\mathrm{P}$ lc à $\mathrm{P} 4$ ) sont régulièrement présents dans les échantillons des Marnes d'Artigueloutan. Dans les Marnes de Gan on a identifié des globotruncanidés du Crétacé et des foraminifères planctoniques du Paléocène et de l'Yprésien basal : Igorina sp., Subbotina triangularis, Acarinina " chascanona", Morozovella gr. angulata.

L'association des foraminifères en provenance d'un niveau marneux des Sables de Guillempau (éch. B4), est variée et caractérisée par la présence de formes agglutinées de la famille Bathysiphonidae. Les foraminifères planctoniques sont rares (quelques dizaines d'exemplaires). Morozovella angulata, $M$. apanthesma, Subbotina triangularis et Parasubbotina varianta sont les plus nombreux, tandis que Igorina albeari n'est représentée que par un seul spécimen. L'absence de Globanomalina pseudomenardii, en combinaison avec la présence de $P$. varianta semble, d'après les données de Berggren et al. (1995, p. 150), indiquer la zone $\mathrm{P} 3 \mathrm{~b}$. La présence du petit benthique Nuttallides truempyi, indique un milieu de dépôt entre 500 et $1000 \mathrm{~m}$ de profondeur. Dans la partie inférieure des Marnes de Latapy (éch. B9) nous n'avons pas retrouvé de foraminifères planctoniques. La microfaune est dominée par les formes agglutinées déjà mentionnées. Les Ammodiscus et Bulimina trinitatensis indiquent un milieu bathyal. La partie sus-jacente du Membre de Latapy (éch. B10) contient plusieurs Globanomalina pseudomenardii et G. chapmani, et quelques rares Acarinina mckannai et Igorina albeari. Elle est attribuable à la zone $\mathrm{P}$ $4 \mathrm{a}$, les espèces spécifiques des sous-zones $\mathrm{P} 4 \mathrm{~b}$ et $4 \mathrm{c}$ étant absentes.

Dans la coupe D de Gan-Rébénacq, la disparition de A. subsphaerica et l'apparition de A. soldadoensis sont séparés par $4 \mathrm{~m}$ de sédiments. Selon la définition originale cet intervalle, entre les échantillons D19 et D24, correspond à la sous-zone $\mathrm{P} 4 \mathrm{~b}$. Ceci signifie que la totalité du Membre du Néez et la moitié inférieure du Membre de Libaros appartiennent à la zone $\mathrm{P} 4 \mathrm{a}$, la partie supérieure de ce dernier aux sous-zones $\mathrm{P} 4 \mathrm{~b}$ et $\mathrm{c}$. L'association de la marne sableuse noirâtre dans la partie supérieure du Membre de Libaros (éch. B38), attribuable à la zone $\mathrm{P} 4 \mathrm{c}$ (deux exemplaires de G. pseudomenardii sont présents; absence de Morozovella velascoensis et $M$. subbotinae), semble moins profond que les autres échantillons du Membre (fréquence de Discorbis sp.).

Les échantillons C1 à C3 sont caractérisés par l'absence de Acarinina mckannai et par la présence de $A$. wilcoxensis et de $S$. ef. linaperta. Ce dernier taxon est reconnu uniquement à Pillardou, ce qui supporte leur attribution à cette unité. C1 et C2 se placent dans la zone à Morozovella velascoensis (P 5). La présence de Globanomalina chapmani dans l'échantillon C3 permet de l'incorporer encore dans la zone P 5. Nous considérons que Globanomalina pseudomenardii, observée dans cet échantillon est remaniée. La microfaune de la partie sableuse, basale du Membre du Pillardou n'a pas été examinée dans la coupe B. L'échantillon composé de sables grossiers, prélevé dans la localité-type (cottage du Pillardou, éch. P 1b) appartient à la zone P 5 (fréquentes $M$. velascoensis) avec quelques exemplaires mal conservés de nummulites. Les petits foraminifères infralittoraux (Discorbis sp. et Rotalia sp.) y sont fréquents. L'échantillon E2 est caractérisé par de rares M. velascoensis et de nombreux Morozovella subbotinae. Ce dernier apparaît au sommet de la zone $P$ 4c. La présence des marqueurs de la zone $P 4$ (rares $G$. pseudomenardii et $M$. apanthesma) dans E2 pourrait être le résultat d'un remaniement ( $R$ dans le Tableau), ce qui rendrait difficile son attribution zonale précise.

Les Marnes d'Artigueloutan de la coupe de GanRébénacq appartiennent à la zone à Morozovella subbotinae s. $s$. de Toumarkine et Luterbacher (1985) ou P 6b de Berggren et al. (1995). M. formosa formosa, marqueur de la sous-zone $\mathrm{P} 6 \mathrm{~b}$, apparaît à partir de l'échantillon E5 et persiste jusqu'au milieu des Marnes de Gan (Le Ten ; $x=377.900 ; y=$ 3106.075). Son absence dans les échantillons E3 et E4 est accidentelle, du fait qu'ils appartiennent déjà à la zone NP 11. Morozovella aragonensis est présente dans les échantillons Toulou $\mathrm{Z}(\mathrm{x}=378.575$; y $=3105.675)$ et le Ten, marquant la zone à $M$. formosa formosa ou $\mathrm{P} 7$. Acarinina pentacamerata, dont l'apparition indique la zone à Morozovella aragonensis de Toumarkine et Luterbacher (1985), a été observée dans les échantillons Toulou b $(x=$ $378.050 ; \mathrm{y}=3105.075)$, le Tunnel $(\mathrm{x}=378.375 ; \mathrm{y}=$ $3106.500)$ et de la tuilerie de $\operatorname{Gan}(\mathrm{x}=378.100 ; \mathrm{y}=$ $3107.200)$. Cette zone inclut la partie sommitale de la zone $\mathrm{P} 7$, ainsi que la totalité de la zone $\mathrm{P} 8$ de Berggren et al. (1995).

Les foraminifères benthiques paléocènes n'ont pas été étudiés. La liste de ceux de l'Yprésien inférieur (échantillons E3-E22) et moyen (Acot- tuilerie de Gan) a été publiée par Sztràkos (2000). La présence constante de Nuttallides truempyi indique des profondeurs supérieures à $500 \mathrm{~m}$ dans les bancs marneux des Membres de Guillempau, de Latapy, du Néez, de Libaros, et dans la partie supérieure du Membre de Pillardou (éch. E2), excepté l'échantillon B38 du Membre de Libaros et l'échantillon P lb du 
TABLEAU 1. - Répartition des foraminifères planctoniques dans la coupe de la route Gan-Rébénacq. Abréviations : r - rare ; $\mathbf{c}-\mathbf{c o m m u n ~ ; ~} \mathbf{f}-$ fréquent ; $\mathbf{R}$ - remaniement. Range chart of the planktonic foraminifera in the Gan-Rébénacq road section. Abbreviations $: r-r a r e ; c-c o m m o n ; f-f r e q u e n t ; R-r e w o r k e d$.

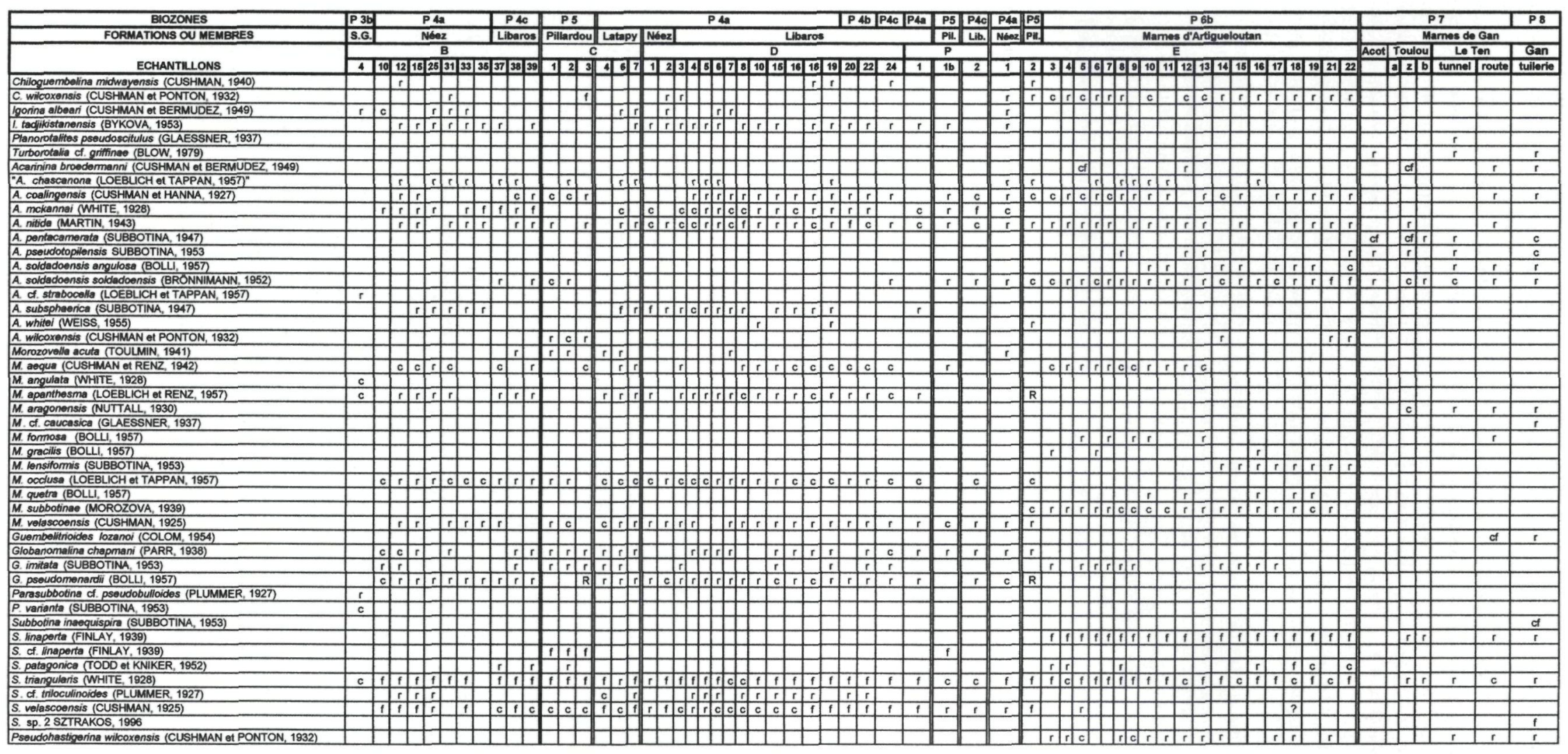


Pillardou. Les intercalations carbonatées et gréseuses, à Orbitoclypeus seunesi et Operculina heberti (B25 et D7) dans les Membres du Néez et de Libaros sont donc des turbidites issues des glissements sousmarins, tout comme les complexes chaotiques reconnus à plusieurs endroits de la coupe B (Fig. 3). En revanche, les figures sédimentaires observées dans le bancs de grès bioclastique $\mathrm{X}$ (passées à laminations obliques et entrecroisées), ainsi que l'aspect des calcaires massifs à la base et au sommet du Membre de Néez montrent que leur milieu de dépôt est l'étage infralittoral.

Certains échantillons des Marnes de Gan sont pauvres en espèces benthiques (entre 1 et 20), comme ceux de Toulou a $(\mathrm{x}=378.600 ; \mathrm{y}=3105.575)$ et $\mathrm{b}(\mathrm{x}$ $=378.050 ; \mathrm{y}=3105.075)$, Bibendot $\mathrm{J}(\mathrm{x}=375.700$; $y=3106.725)$, Laroque $(x=375.750 ; y=3107.125)$, Lapeyre $2(\mathrm{x}=377.400 ; \mathrm{y}=3105.800)$, Acot $(\mathrm{x}=$ $378.800 ; \mathrm{y}=3105.050)$, Bouchou haut $(\mathrm{x}=375.400$; $y=3107.150$ ). Dans ces faunes, les formes infralittorales prédominent : grands foraminifères, miliolidés, Asterigerina bartoniana, Pararotalia spinigera. Les milieux infralittoraux profonds et circalittoraux sont représentés par les échantillons Bibendot I et le Ten, avec Alveolina oblonga, Assilina placentula, Operculina karreri, Planispirillina striatogranulosa, Pentellina pseudosaxorum, Bolivina striatocarinata dans le premier et Dorothia traubi, Pseudogaudryina africana, Oridorsalis umbonatus dans le deuxième. Le nombre d'espèces est de 70 et 60 respectivement. Le milieu le plus profond atteint dans les Marnes de Gan de la région-type correspond à l'étage épibathyal, observé dans les échantillons Toulou $\mathrm{Z}$ et le Tunnel. Dorothia traubi, Reticulophragmium ef. rotundidorsatum, Martinottiella cocoaensis, Drepaniota ef. lorifera, Osangularia pteromphalia font partie de ces associations, composées de 34 à 47 espèces. Les milieux infralittoraux réapparaissent au sommet des Marnes de Gan, mais avec une faune plus riche qu'à sa base. La microfaune des échantillons prélevés par Nolf (IRScNB, Bruxelles) dans la marnière de Gan comporte 57 espèces avec de nombreux grand foraminifères, Adelosina longirostra, Pentellina pseudosaxorum, Asterigerina bartoniana, Cuvillierina yarzai, Civrieuxia palmerae, Ganella neumannae, Neorotalia tuberculata et d'autres.

\section{CALIBRATION DES ZONATIONS UTILISÉES}

L'étude des foraminifères et des nannofossiles calcaires que nous venons de présenter ici repose sur la même série d'échantillons, permettant une calibration précise des diverses biozonations adoptées, et une comparaison avec modèle biostratigraphique standard de Berggren et al. (1995). Notre étude montre que la limite des sous-zones $\mathrm{P} 4 \mathrm{a} / \mathrm{b}$ se trouve incontestablement au sein de la zone NP 8, comme indiqué dans le schéma standard. Par contre, la limite $\mathrm{P}$ 4b/c semble coincider avec la limite NP 8/NP 9 en Aquitaine, ou à peine la surpasser jusque dans la base de NP 9 (les deux options sont possibles les foraminifères de D23 n'ayant pas été analysés). En ce point nos données diffèrent du schéma général, dans lequel cette limite est inclue dans la zone NP 8 . Ce désaccord est vraisemblablement dû au fait que le marqueur A. soldadoensis apparaît un peu plus tard en Aquitaine. Nos propres recherches sont conformes à celles de Berggren et al. pour ce qui est la position de la limite $\mathrm{P}$ 4/P 5. Celle-ci se situe dans la zone NP 9. Les autres limites des biozones n'ont pas pu être précisées faute des affleurements trop discontinus, et sont donc difficiles à juxtaposer. L'échantillon $\mathbf{B} 4$, rattaché à la zone $\mathbf{P} 3 \mathbf{b}$ contient une association de nannofossiles typique de la partie supérieure de la zone NP 4, ce qui n'est pas en contradiction avec Berggren et al. Les échantillons $\mathrm{C} 3$ et E2, dont les foraminifères suggèrent une partie élevée de la zone $\mathrm{P} 5$, sont sous réserve attribués à la base de la zone NP 10, le Membre d'Artigueloutan (E3 jusque E22) aux zones P 6b et NP 11, et les Marnes de Gan aux zones $\mathrm{P}$ 7-8 et NP 12-13. Ces résultats ne contredisent non plus les corrélations établies par Berggren et al. (1995). Les principaux événements biostratigraphiques recensés dans le Paléogène de la région de Gan sont rassemblés dans la Figure 5.

\section{REMARQUES SUR L'INTERPRÉTATION SÉQUENTIELLE}

L'histoire séquentielle du Paléogène aquitain (Sztràkos et al., 1997 ; Gély et Sztràkos, 2000) fut reconstruite à partir des coupes d'une centaine de forages (diagraphies et échantillons) et d'affleurements à travers tout l'Aquitaine méridionale. La coupe de la route Gan-Rébénacq, largement exposée et assez complète, permet de tester ce modèle, en essayant de reconnaître les diverses séquences de dépôt de troisième ordre et de préciser leur âge (Fig. 6). Cette coupe s'avère parmi les meilleures d'Aquitaine, malgré que certaines limites de séquences restent cachées sous la végétation.

Les difficultés de comparaison avec des autres coupes viennent du fait que les faciès changent entre 


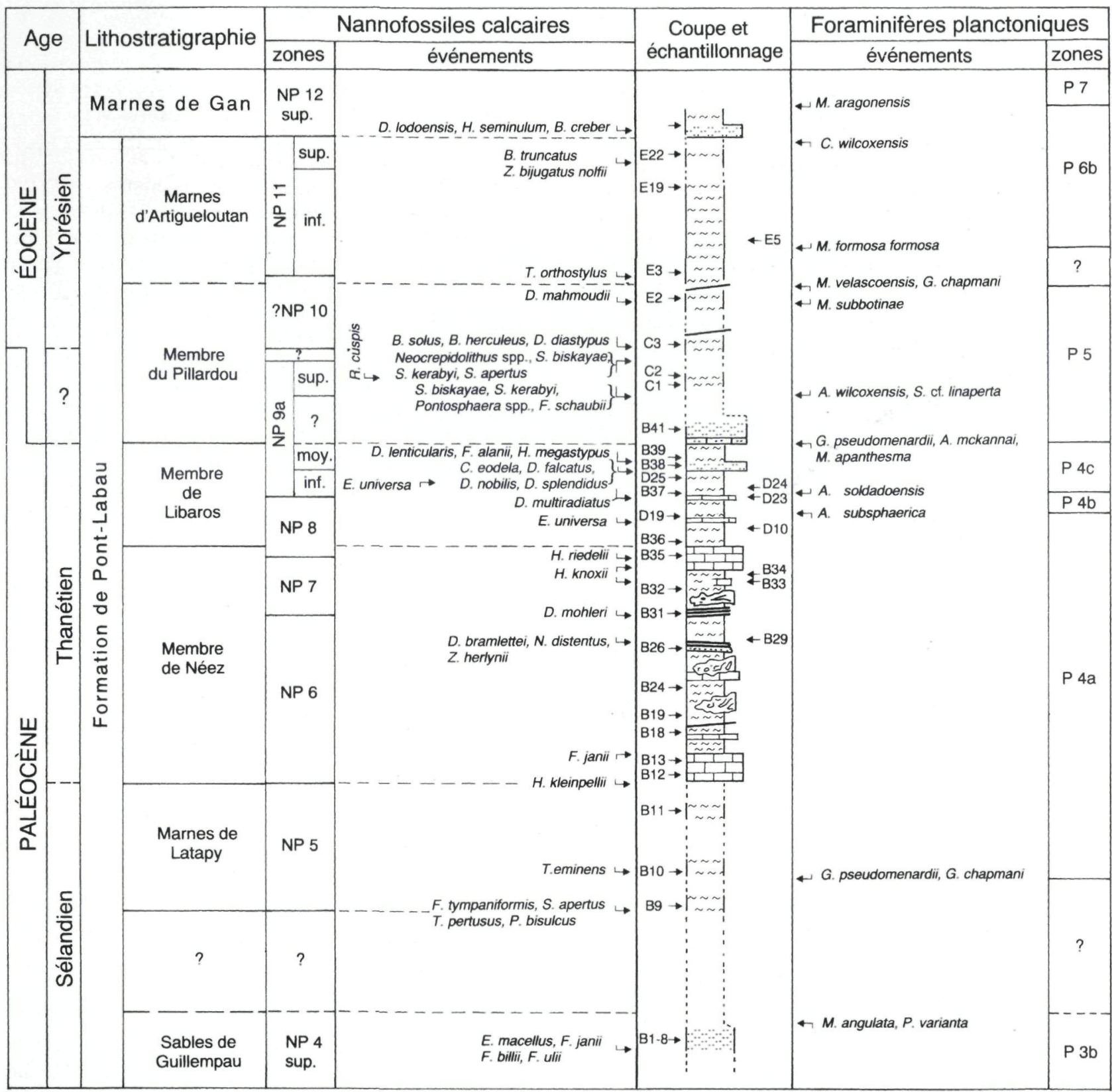

FIG. 5. - Les principaux événements à nannofossiles calcaires et à foraminifères planctoniques dans le Paléogène inférieur de la coupe de la route Gan-Rébénacq.

The major calcareous nannofossil and planktonic foraminiferal events in the Lower Paleogene of the Gan-Rébénacq road section.

les affleurements du sud et les sondages plus au nord. Ainsi, la région de Gan est fortement soumis à l'arrivée des élements grossiers, turbiditiques et au nord, on observe l'influence croissante d'une plate- forme carbonatée qui occupe la partie septentrionale du Bassin de l'Adour. Par conséquent, le découpage biostratigraphique fin offert par les nannofossiles calcaires n'y est plus possible et on doit utiliser les 
W COUPES RÉGION DE GAN

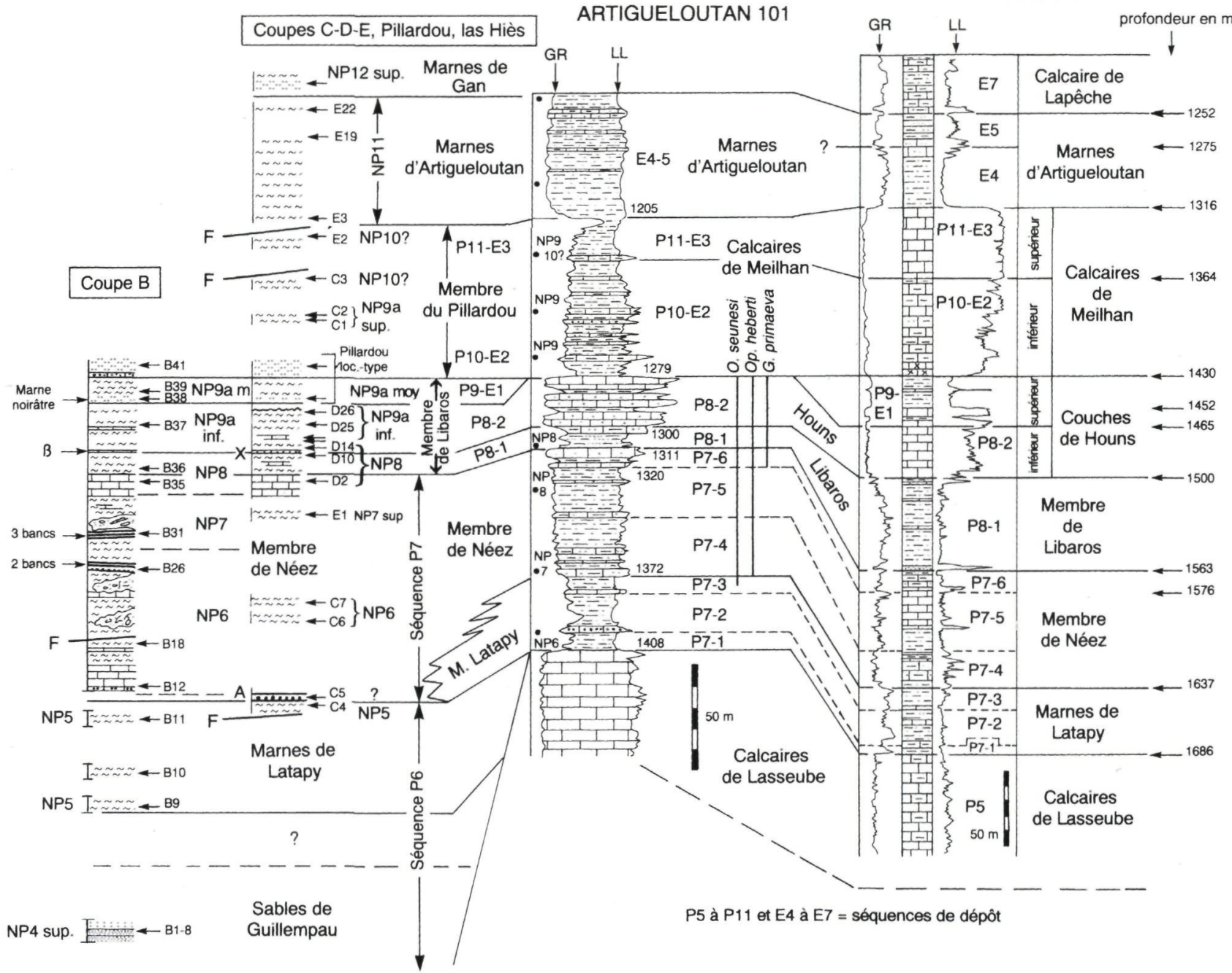

FIG. 6. - Stratigraphie intégrée du Paléogène inférieur de la région de Gan et corrélations avec les coupes de référence d'Artigueloutan 101 et de Libaros 1, situées plus à l'est (NP = zones à nannofossiles; $\mathrm{P} 5$ à $\mathrm{P} 11$ et $\mathrm{E} 4$ à $\mathrm{E} 7$ = séquences de dépôt).

Integrated stratigraphy of the Lower Paleogene of the Gan area and correlations with the Artigueloutan 101 and Libaros 1 reference sections, located further east (NP = nannofossil zones; P5 to P11 and E4 to E7 = depositional sequences).

grands foraminifères (Orbitoclypeus, Alveolinidae) donnant une sensibilité moindre, problème auquel s'ajoutent des difficultés liées à la corrélation des biozones issues de plusieurs groupes. Pour cela, nous avons utilisé le schéma proposé par Hardenbol et al. (1998).

Notre analyse montre que les Sables de Guillempau et les Marnes de Latapy constituent une seule séquence de dépôt de troisième ordre, appelée P6 dans Sztràkos et al. (1997). Les Sables de Guillempau sont interprétés comme prisme de bas-niveau, les Marnes de Latapy sus-jacentes comme prisme de haut niveau. On n'a pas pu observer la base de cette séquence, mais d'après Sztràkos et al., elle semble débuter dans la partie supérieure de la zone NP 4 . Cela correspond à nos propres recherches, vu que notre premier échantillon B1 dans les Sables de Guillempau appartient au sommet de NP 4. Le sommet de la séquence se rattache à la zone NP 5 , si le calcaire gréseux A représente la base du Membre de Néez. Dans le cas, où ce calcaire représente un niveau grossier dans les Marnes de Latapy, la partie supérieure 
de ces marnes pourraient déjà appartenir à la zone NP 6. Le Membre de Néez, qui comprend les zones NP 6, NP 7 et en partie la zone NP 8, définit une nouvelle séquence, correspondant en partie à la séquence P7 de Sztràkos et al. Cette séquence est marquée par des changements paléogéographiques. A la suite de la déformation du sillon nord-pyrénéen, une plate-forme carbonatée apparaît à la base du Membre de Néez à Gan et des marnes bathyales de Latapy équivalentes progradent sur la surface érodée des Calcaires de Lasseube plus au nord, dans le puits Artigueloutan 101 (Fig. 6). Au-dessus de cet intervalle transgressif s'installe un système turbiditique épibathyal à Gan, et une alternance de calcaire et d'argile plus au moins sableux à Artigueloutan. Une barre calcaire infralittorale surmonte cet ensemble dans les deux régions.

La base du Membre de Libaros, caractérisée par des sables grossiers dans le forage de Libaros 1 (voir Sztràkos et al., 1997, p. 33) et considérée comme limite de la paraséquence P7.4 par Sztràkos et al. (1997), est interprétée ici comme le début de la séquence P8. Dans cette région les argiles sableuses à glauconie, surmontant cette base sableuse annoncent l'approfondissement du milieu de dépôt et une nouvelle transgression. La base de cette séquence P8 redéfinie, qui comprend la partie supérieure de la zone NP 8 et la base de NP 9, n'est pas représentée par un contact érosif, ni de sables grossiers dans la coupe de Gan-Rébénacq. On n'a vu qu'une forte augmentation en Braarudosphaera alta et B. bigelowii, formes d'eau côtières, à la base de la coupe D (D1) dans la transition du Membre du Néez au Membre de Libaros. En revanche, leur limite est nette dans le puits Artigueloutan 101. Le contact érosif au milieu du Membre de Libaros à la base du grès conglomératique $\mathrm{X}$ est considéré comme limite de paraséquence (entre P8-1 et P8-2) et non comme la base d'une turbidite, vu ses ressemblances avec le grès basal du Membre de Pillardou et la présence de grains de glauconie. La couche sableuse noirâtre à foraminifères benthiques infralittoraux et riche en matière organique (B38) reflète le retour à des conditions plus côtiers. Sa base est interprétée comme limite de séquence. Celle-ci se rattache vraisemblablement à la séquence P9-E1 de Sztràkos et al. La partie inférieure du Membre de Pillardou, comprenant le grès grossier basal, les sables et marnes sus-jacentes reconnus dans le sommet de la coupes $\mathrm{B}$ et la base de la coupe $\mathrm{C}$ ( $\mathrm{C} 1$ et $\mathrm{C} 2$ ) représente la séquence $\mathrm{P} 10-\mathrm{E} 2$ de Sztràkos et al. Sa portion supérieure, dont on ne connaît qu'une zone marneuse (C3 et E2), appartiendrait à la séquence P11-E3. Le contact avec les Marnes d'Artigueloutan se situe dans une zone mal exposée. Le premier échantillon que nous avons pu étudier de ces marnes (E3) se rattache déjà à la base de la NP 11, le dernier (E22) à une portion élevée de la NP 11 (sous-zone III al de Steurbaut, 1991). La partie inférieure pourrait appartenir à la séquence E5, la partie supérieure à la séquence suivante. La base des Marnes de Gan, representée par des sables fins, se rattache à la base de la partie supérieure de la NP 12, et représente la séquence E7. Les couches de la tuilerie et de Bosdarros, qui chevauchent la limite NP 12 - NP 13, appartiennent à la séquence E8.

\section{CONSIDÉRATIONS CHRONOSTRATIGRAPHIQUES}

La comparaison des données stratigraphiques de la coupe de la route Gan-Rébénacq avec celles du Bassin de la Mer du Nord (Steurbaut, 1998) et de la Tunisie (Steurbaut et al., 2000) suggèrent que les Sables de Guillempau et les Marnes de Latapy de la coupe-type, appartiennent au Sélandien. Le Membre de Néez et le Membre de Libaros représentent le Thanétien (zone NP 6 jusqu'à la partie médiane de la zone NP 9).

La position de la limite Paléocène/Eocène est actuellement sujette à discussion (Schmitz et al., 2000). D'après les recommandations de la "International Subcommission on Paleogene Stratigraphy », basées sur un vote parmi ses membres, la base de l'excursion négative du $\delta^{13} \mathrm{C}$, et pas le maximum, ni le sommet, est le critère pour la définition de la limite $\mathrm{Pa}$ léocène/Eocène (Luterbacher et al., 2000 ; Schmitz, 2000). Les corrélations entre les données isotopiques et les données micropaléontologiques (nannofossiles calcaires et foraminifères benthiques) établies dans plusieurs coupes méditerranéennes (en Egypte, Espagne, Italie) suggèrent qu'en Aquitaine le maximum de l'excursion négative $\mathrm{du} \delta^{13} \mathrm{C}$ se situe au sein du Membre du Pillardou. Cela implique que la partie supérieure du Membre du Pillardou se rattacherait à l'Eocène. La position de sa partie inférieure reste difficile à préciser, faute de l'absence d'études isotopiques en Aquitaine. Dans le bassin de la Mer du Nord, la base de l'excursion négative du $\delta^{13} \mathrm{C}$ coincide avec la base de la séquence Y-1 qui est associée à un abaissement majeur du niveau marin (Steurbaut, 1998 ; Magioncalda et al., 2001). Les changements sédimentologiques et micropaléontologiques qui se sont produits à la base du Membre du Pillardou suggèrent son équivalence avec la base de la séquence $\mathrm{Y}$ 1. Tenant compte de ces données il n'est pas exclu que la base du Membre du Pillardou coinciderait avec la base de l'excursion négative du $\delta^{13} \mathrm{C}$ et, par 
définition, correspondrait à la limite Paléocène/Eocène. Dans ce cas, cette limite serait proche de celle des zones à Glomalveolina levis/Alveolina cucumiformis et de la base de la zone à Nummulites fraasi.

Les Marnes de Artigueloutan et les Marnes de Gan sont d'âge yprésien. L'abondance de remaniements dans la nannoflore et la faune des foraminifères indique d'importants mouvements tectoniques dans la region de Gan-Rébenacq pendant l'Yprésien.

\section{CONCLUSIONS}

Le levé stratigraphique détaillé de plusieurs coupes le long de la route Gan-Rébénacq a permis de reconstituer l'évolution des corps sédimentaires au sein de la Formation de Pont-Labau et de délimiter les divers membres. L'intégration des données micropaléontologiques a démontré que la série paléocène et éocène inférieur y est relativement complète, mais compartimentée par plusieurs failles majeures. Les divers membres de la Formation de Pont-Labau, ainsi que les Marnes de Gan sus-jacentes ont pu être datés en appliquant la zonation à nannofossiles calcaires NP de Martini (1971) et celle à foraminifères planctoniques $\mathrm{P}$ de Berggren et al. (1995). En dehors du Membre d'Artigueloutan dont la signification séquentielle reste à préciser, ces membres s'organisent en sept séquences de dépôt (P6, P7, P8-1, P8-2, toutes redéfinies par rapport aux travaux de Sztràkos et al., 1997 ; P9-E1, P10-E2 et P11-E3). Le passage paléocène-éocène, représenté par le Membre du Pillardou et le passage de la Formation de Pont-Labau aux Marnes de Gan sont assez mal exposés et restent à préciser. La calibration des zonations micropaléontologiques utilisées dans le cadre de ce travail a révélé que le modèle biostratigraphique standard de Berggren et al. est applicable dans le domaine aquitain, à une exception près, vu qu'en Aquitaine la limite $\mathrm{P} 4 \mathrm{~b} / \mathrm{c}$ ne tombe pas au sein de la zone NP 8, mais coincide avec la limite NP 8/NP 9. En outre, il en est ressorti que la séquence des événements biotiques survenus dans les zones NP 11 et NP 12 de l'Yprésien du Bassin de la Mer du Nord (Steurbaut, 1991 et Steurbaut dans Schmitz et al., 1996) est également reconnaissable dans l'Aquitaine méridionale.

Les datations précises documentées dans la présente note fournissent une meilleure compréhension de la complexité de l'histoire sédimentaire de la région de Gan. Celle-ci comprend plusieurs étapes. Après la phase tectonique fini-danienne, engendrant une forte érosion, la subsidence régionale reprend avec l'accumulation des Sables de Guillempau, dépôt turbiditique appartenant au sommet de NP 4 et à la $\mathrm{P} 3 \mathrm{~b}$ et des Marnes de Latapy de milieu bathyale, attribuable à la NP 5 et la $\mathrm{P} 4 \mathrm{a}$. Ces dépôts constituent une seule séquence de dépôt, appelée P6. On n'a pas pu éclaircir les événements survenus au passage des séquences $\mathrm{P} 6$ et $\mathrm{P} 7$ à cause de la végétation. Toutefois, la nature de la barre calcaire basale du Membre de Néez témoigne d'une forte régression dans la région. Cette barre correspond à un prisme de bas-niveau marin. Elle se rattache à la zone NP 6 et est surmontée par une alternance de marnes et bancs calcaires, marquant un approfondissement de la tranche d'eau. La barre calcaire supérieure du Membre de Néez chevauche la limite NP 7/NP 8. Elle annonce le retour de dépôts de plate-forme. Le base du Membre de Libaros, grossière et glauconieuse dans le sondage de Libaros (Sztràkos et al., 1997) est interprêtée comme base de la nouvelle séquence P8 redéfinie ici. Dans la région de Gan, se situant plus loin du rivage cette base n'est pas si clairement exprimée et ne correspond qu'à un changement de faciès carbonatés aux faciès marneux. La succession des événements dans les associations des nannofossiles calcaires du Membre de Libaros (la série d'apparitions et disparitions dans les zones NP 8 et NP 9) suggère une sédimentation quasi continue en milieu circalittoral-bathyal. Toutefois, l'apparition d'une surface d'érosion et de la glauconie à la base du grès $X$ et d'une marne sableuse, riche en matière organique, un peu plus haut, témoignent de deux épisodes de régression au sein de ce membre. Le premier événement est considéré comme limite de paraséquence (P8-2) et le deuxième est interprêté comme base de séquence de dépôt P9-E1. Les Marnes de Cassoua bathyales de la zone NP8, bien développées entre Peyrehorade et Orthez, ne sont plus représentées dans la région de Gan, mais sont remplacées par la partie inférieure du Membre de Libaros.

Le plus grand abaissement du niveau marin dans la région de Gan s'est produit à la limite du Membre de Libaros circalittoral à bathyal au Membre du Pillardou infralittoral. Celle-ci représente la base de la séquence P10-E2. D'après les associations de nannofossiles, aucune séquence de dépôt semble manquer à ce niveau, n'indiquant donc qu'une courte phase d'érosion ou de non-dépôt. La suite de la série affleure assez mal, ne laissant que des informations fragmentaires. On a observé la partie supérieure de la sous-zone NP 9a et vraisemblablement la zone NP 10 dans le Membre du Pillardou, tandis que les Marnes d'Artigueloutan semblent débuter à la base de la zone NP 11. Les sables fins glauconieux à la base des Marnes de Gan annoncent une nouvelle transgression, datée de la base de la partie supérieure de la zone NP 12. Celle-ci a été précédée par 
une phase tectonique importante, vu l'abondance des nannofossiles et foraminifères remaniés dans les assemblages des Marnes de Gan. Ces sables sont surmontés par des marnes circalittorales-bathyales, représentant les Marnes de Gan, atteignant une épaisseur de plusieurs centaines de mètres. A cette époque, la région de Gan connaît une subsidence maximale.

Les changements de lithofaciès et les grandes discontinuités dans la sédimentation du Cénozoïque inférieur de la région de Gan sont comparables et contemporains à ceux observés dans les zones méridionales du Bassin de la Mer du Nord (Knox, 1996 ; Steurbaut, 1998), suggérant d'importantes phases de surrection aux frontières de la plaque ouest-européenne : collision dans la région pyrénéenne et montée de la plume mantellique proto-Islandaise en dessous de Groenland.

\section{REMERCIEMENTS}

Les auteurs tiennent à remercier D. Nolf (IRScNB) pour l'assistance lors du levé de la coupe, ainsi que H. Depotter, J. Cilis et W. Miseur pour leur collaboration dans la réalisation des figures et des planches. Nous exprimons également notre reconnaissance au rapporteur $K$. von Salis et au rapporteur anonyme pour leurs suggestions et corrections qui ont grandement contribué à l'amélioration du manuscrit. Ce travail a été réalisé avec le soutien de l'Institut Royal des Sciences Naturelles de la Belgique et du Fonds National de la Recherche Scientifique de la Belgique (bourse FRFC-IC 2.4540.99).

\section{BIBLIOGRAPHIE}

Aubry M.-P. (1999) : Handbook of Cenozoic Calcareous Nannoplankton. Book 5 : Heliolithae (Zygoliths and Rhabdoliths). Micropaleontology handbook series, Micropaleontology Press : American Museum of Natural History, 368 p.

Aubry M.-P., Berggren W.A., Cramer B.S., Dupuis C., Kent D.V., Ouda K., Schmitz B. et Steurbaut E. (1999) : Paleocene/Eocene boundary sections in Egypt. In Aubry M.-P., Ouda K. et Berggren W.A. (eds) : Late Paleocene-Early Eocene events from North Africa to the middle East, The First International Conference of the Geology of Africa (Nov. 23-25 , 1999 - Assiut, Egypt), p. 1-11.

Aubry M.-P., Cramer B.S., Miller K.G., Wright J.D., Kent D.V. et OLsson R.K. (2000) : Late Paleocene event chronology : Unconformities not diachrony. Bull. Soc. géol. France, Paris, t. 171, $\mathbf{n}^{\circ} 3$, p. 367-378.

Backman J. et SHACKLETON N.J. (1983) : Quantitative biochronology of Pliocene and Early Pleistocene calcareous nannofossils from the Atlantic, Indian and Pacific Oceans, Micropaleonto$\log y$, vol. 8, $\mathbf{n}^{\circ} 2$, p. 141-170.

Berggren W.A, Kent D.V., Swisher C.C. III et Aubry M.-P. (1995) : A revised Cenozoic geochronology and chronostratigraphy. In Berggren W.A., Kent D.V., Aubry M.-P. et Hardenbol J. (eds): Geochronology, time scales and global stratigraphic correlation. SEPM Spec. Publ., Tulsa, vol. 54, p. 129-212.

Berggren W. A. et Norris R. N. (1997) : Biostratigraphy, phylogeny and systematics of Paleocene trochospiral planctic foraminifera. Micropaleontology, New York, vol. 43, suppl. 1, p. 1-116.

BIGG P.J. (1982) : Eocene planktonic foraminifera and calcareous nannoplankton from the southern Aquitaine Basin, France. Rev. Esp. Micropal., Madrid, vol. XIV, p. 367-400.

Cavelier C., Fries G., Lagarigue J.-L. et Capdeville J.-P. (1997) : Sédimentation progradante au Cénozoïque inférieur en Aquitaine méridionale : un modèle. Géol. France, Ed. B.R.G.M. et S.G.F., Orléans, $n^{\circ} 4$, p. 69-79.

DAGUIN F. (1948) : L'Aquitaine occidentale. In De Lapparent, A.F. (Dir.) : Géologie régionale de la France, Paris, V, 232 p.

DouvILLÉ H. (1919) : L'Éocène inférieur en Aquitaine et dans les Pyrénées. Mém. Explic. Carte géol. dét. France, 84 p.

Flicoteaux R. (1967) : Contribution à l'étude stratonomique du Paléocène du Sud-Ouest Aquitain. Relations entre les caractères stratonomiques et les rythmes enregistrés par les diagraphies. Thèse Doct., Univ. de Bordeaux, 151 p. et annexe $91 \mathrm{p}$ (inédites).

Flicoteaux R. (1972) : L'analyse stratonomique, méthode d'études des différents faciès du flysch paléocène de Pau et de leur milieu de dépôt. Application aux problèmes posés par les corrélations entre affleurements et sondages. In Colloque sur les méthodes et tendances de la stratigraphie, Orsay, septembre 1970. Mém. B.R.G.M., Paris, $\mathrm{n}^{\circ}$ 77, p. 615-630.

Fondecave-Wallez M.J., Eichène P. et Peybernès B. (1999) : La limite Crétacé-Tertiaire et le Paléocène "inférieur " de la region de Nay (Béarn). Bull. Soc. Hist. Nat. Toulouse, vol. 135, p. 9-20.

GÉLY J.-P. et SzTrÀKos K. (2000) : L'évolution paléogéographique et géodynamique du Bassin aquitain au Paléogène : enregistrement et datation de la tectonique pyrénéenne. Géol. France, Ed. B.R.G.M. et S.G.F., Orléans, $\mathbf{n}^{\circ} 2$, p. 31-57.

Gubler Y. et Pomeyrol R. (1946) : Nouvelles observations stratigraphiques dans l'Eocène au sud de Pau (Basses-Pyrénées). Bull. Soc. géol. France, Paris, t. 16, n⿳0 5, p. 423-444.

Hardenbol J., Thierry J., Farley M.B, Jaceuin T., de Graciansky P.-C. et VaIl P.R. (1998) : Mesozoic and Cenozoic sequence chronostratigraphic framework of European Basins. In de Graciansky P.-C., Hardenbol J., Jaquin T. et Vail P.R. (eds) : Mesozoic and Cenozoic Sequence Stratigraphy of European Basins. SEPM, Society for Sedimentary Geology, Spec. Pub., Tulsa, Oklahoma, no 60 , p. 3-15.

HAY W.W.(1964) : Utilisation stratigraphique des Discoastéridés pour la zonation du Paléocène et de l'Eocène inférieur. In Colloque sur le Paléogène (Bordeaux, Septembre 1962). Mém. Bur. Rech. Géol. Min., Ed. B.R.G.M., Paris, tome II, p. 885889.

HAY W.W et MoHLER H.P. (1967) : Calcareous nannoplankton from Early Tertiary rocks at Pont Labau, France and Paleocene-Early Eocene correlations. Journ. Paleont., Lawrence, vol. $41, \mathrm{n}^{\circ} 6$, p. 1505-1541.

Kapellos C. et Schaub H. (1973) : Zur Korrelation von Biozonierungen mit Grossforaminiferen und Nannoplankton im Paläo- 
gen der Pyrenäen. Eclog. Geol. Helv., Basel, vol. 66, no 3 , p. 687-737.

KNox R.W. O'B. (1996) : Tectonic controls on sequence development in the Palaeocene and earliest Eocene of southeast England : implications for North Sea stratigraphy. In Hesselbo, S.P. et Parkinson, D.N. : Sequence Stratigraphy in British Geology. Spec. Publ. Geol. Soc. London, London, $n^{\circ}$ 103, p. 209-230.

LEZAUD L. (1964) : Essai d'utilisation de quelques nannofossiles (Coccolithophoridés et groupes annexes) en stratigraphie de l'Eocène d'Aquitaine occidentale. In Colloque sur le Paléogène (Bordeaux, Septembre 1962). Mém. Bur. Rech. Géol. Min., Ed. B.R.G.M., Paris, tome I, p. 289-294.

Lezaud L. (1967) : Contribution à l'étude des Nannofossiles calcaires de l'Eocène (Coccolithophoridés). Leur rôle stratigraphique en Aquitaine sud-ouest (Chalosse, Béarn, Bas-Adour). Thèse Doct., Univ. de Paris, 238 p., 25 pl. (inédite).

LEZAUD L. (1968) : Espèces nouvelles de Nannofossiles calcaires (Coccolithophoridés) d'Aquitaine sud-ouest. Rev. Micropaléont., Paris, vol. 11, $\mathrm{n}^{0} 1$, p. 22-28.

Luterbacher H.P., Hardenbol J. et Schmitz B. (2000) : Decision of the Voting Members of the International Subcommission on Paleogene Stratigraphy on the Criterion for the Recognition of the Paleocene/Eocene Boundary. International Subcommission on Paleogene Stratigraphy, Newsletter $\mathbf{n}^{0}$ 9, p. 13.

Magioncalda R., Dupuis C. et Steurbaut E. (2001) : Organic carbon isotopic changes $\left(\left({ }^{13} \mathrm{Corg}\right)\right.$ in lagoonal Paleocene/Eocene boundary settings in Northern Belgium. In Ash A.W. et Wing S. L. (eds) : Climate and Biota of the Early Paleogene. Abstracts volume, International meeting July 3-8, 2001, Powell, Wyoming, p. 60.

Martini E. (1961) : Nannoplankton aus dem Tertiär und der obersten Kreide von SW-Frankreich. Senck. leth., Frankfurt am Main, Band 42, $\mathrm{n}^{\circ} 1 / 2$, p. 1-41.

Martini E. (1971) : Standard Tertiary and Quaternary calcareous nannoplankton zonation. Proceedings 2d Planktonic Conference (Roma, 1970), Ed. Technoscienza, vol. 2, p. 739-785.

Molina E., Arenillas I. et Pardo A. (1999) : High resolution planktic foraminiferal biostratigraphy and correlation across the Paleocene/Eocene boundary in the Tethys. Bull. Soc. géol. France, Paris, t. $170, \mathrm{n}^{0} 4$, p. 521-530.

Monechi S., Angori E. et Speijer R.P. (2000a) : Upper Paleocene biostratigraphy in the Mediterranean region : Zonal markers, diachronism, and preservational problems. In Schmitz B., Sundquist B. et Andreasson F.P. (eds) : Early Paleogene Warm Climates and Biosphere Dynamics. GFF, Stockholm, vol. 122, part 1, p. 108-110.

Monechi S., Angori E. et Von Salis K. (2000b) : Calcareous nannofossil turnover around the Paleocene/Eocene transition at Alamedilla (southern Spain). Bull. Soc. géol. France, Paris, t. $171, \mathrm{n}^{\circ} 4$, p. $477-489$.

O'Gorman G. (1923) : Le gisement cuisien de Gan (Basses Pyrénées). Pau, p. I-XXV.

Olsson R.K., Hemleben C., Berggren W. A. et Huber B. T. (1999) : Atlas of Paleocene planktonic foraminifera. Smithsonian Contr. Paleobiol., Washington, 85, 252 p.

Perch-Nielsen K. (1985) : Cenozoic calcareous nannofossils. In Bolli H.M., Saunders J.B. et Perch-Nielsen K. (eds) : Plankton Stratigraphy. Cambridge Earth Science Series, Cambridge, vol. 11, p. 427-554.

Peybernès B., Fondecave-Wallez M.J. et Eichène P. (1996) : Sur l'âge paléogène de certaines « lames triasiques " nord- et sous-pyrénéennes. Bull. Soc. géol. France, Paris, t. 167, nº 6 , p. 761-771.
Plazlat J. C., Toumarkine M. et Villatte J. (1975) : L'âge des calcaires pélagiques et néritiques de la base du Tertiaire (Danien, Paléocène), Bassin basco-cantabrique et béarnais (Espagne, France). Mise au point sur leur faunes d'Echinides. Eclog. geol. Helv., Basel, vol. 68, n⿳0 3, p. 614-647.

Rovern A.J.T. (1979) : Lineages in Early Paleogene Calcareous Nannoplankton. Utrecht Micropal. Bull., Hoogeveen, $\mathbf{n}^{\circ} 22$, $231 \mathrm{p}$.

Schmitz B. (2000) : Is the CIE in the late Paleocene or the early Eocene? International Subcommission on Paleogene Stratigraphy, Newsletter $\mathbf{n}^{\circ}$ 9, p. 19.

Schмiтz B., Aubry M.-P. et ZAchos J. (2000) : Preface \& Summary. In Schmitz B., Sundquist B. et Andreasson F.P. (eds) : Early Paleogene Warm Climates and Biosphere Dynamics. GFF, Stockholm, vol. 122, part 1, p. 4-6.

Schmitz B., Hellmann-Clausen C.. King C.. Steurbaut E., Andreasson F.P., Corfield R.M. et Cartlidge J.E. (1996) : Stable isotope and biotic evolution in the North Sea during the early Eocene : the Albaek Hoved section, Denmark. In Knox R. W. O'B., Corfield R.M. et Dunay R.E. (eds) : Correlation of the Early Paleogene in Northwest Europe. Spec. Publ. Geol. Soc. London, London, 101, p. 275-306.

SEYVE Ch. (1984) : Etude micropaléontologique du passage Crétacé/Tertiaire, du Paléocène et de l'Yprésien au sud de Pau. Mém. Sci.Terre, Université Paris VI, no 84-11, 187 p.

Steurbaut E. (1991) : Ypresian calcareous nannoplankton biostratigraphy and palaeogeography of the Belgian Basin. In Dupuis C., De Coninck J. et Steurbaut E. (eds) : The Ypresian stratotype. Bull. Soc. belge Géol., Bruxelles, vol. 97, $\mathrm{n}^{\circ} 3-4$ (1988), p. 251-285.

Steurbaut E. (1998) : High-resolution holostratigraphy of Middle Paleocene to Early Eocene strata of Belgium and adjacent areas. Palaeontographica, Stuttgart, Abt. A, Bd. 247, n 5-6, p. $91-156$.

Steurbaut E., Dupuis C., Arenillas I., Molina E. et Matmati M.F. (2000) : The Kalaat Senan section in Central Tunisia : a potential reference section for the Danian/Selandian boundary. In Schmitz B., Sundquist B. et Andreasson F.P. (eds) : Early Paleogene Warm Climates and Biosphere Dynamics. GFF, Stockholm, vol. 122, part 1, p. 158-160.

SzTRÀKos K. (1996) : Le Thanétien supérieur et l'Yprésien du Bassin de l'Adour (Aquitaine, france). Stratigraphie et paléoenvironnement. Etude des foraminifères. Cah. Micropal., CNRS éd., Paris, n. sér., vol. 10, $\mathbf{n}^{\circ} 1$, p. 25-63.

Sztrìkos K. (2000) : Les foraminifères de l'Eocène du Bassin de l'Adour (Aquitaine, France) : biostratigraphie et taxinomie. Rev. Micropaléont., Paris, vol. 43, $\mathrm{n}^{0}$ 1-2, p. 71-172.

Sztràkos K., Gély J.P., Blondeau A. et Müller C. (1997) : Le Paléocène du Bassin sud-aquitain : lithostratigraphie, biostratigraphie et analyse séquentielle. Géol. France, Ed. B.R.G.M. et S.G.F., Orléans, $\mathrm{n}^{0} 4,27-54$.

Sztràkos K., Gély J.P., Blondeau A. et Müller C. (1998) : L’Eocène du Bassin sud-aquitain : lithostratigraphie, biostratigraphie et analyse séquentielle. Géol. France, Ed. B.R.G.M. et S.G.F., Orléans, $n^{\circ} 4$, p. 57-105.

Toumarkine M. et Luterbacher H.P. (1985) : Paleocene and Eocene planktic foraminifera. In Bolli H.M., Saunders J.B. et Perch-Nielsen K. (eds) : Plankton Stratigraphy. Cambridge Earth Science Series, Cambridge University Press, vol. 1, 5, p. $87-154$.

van Morkhoven F.C.P.M., Berggren W.A. et Edwards A.S. (1986) : Cenozoic cosmopolitan deep-water benthic Foramini- 
fera. Bull. Centres Rech. Expl.-Prod. Elf-Aquitaine, Mém. 11, $421 \mathrm{p}$.

VAROL O. (1989) : Palaeocene calcareous nannofossil biostratigraphy. In Crux J.A. et Van Heck S.E. (eds) : Nannofossils and their applications. British Micropal. Soc. Ser., chap. 12, p. 267-310.

VAROL O. (1992) : Sullivania a new genus of Palaeogene coccoliths. Journ. Micropal., vol. 11, $\mathrm{n}^{\circ}$ 2, p. 141-149.

von Salis K., Ouda K., SaAd El Din M., Tantawy A.A. et Bernasconi S. (1998) : Calcareous Nannofossils, Foraminifera and stable isotope studies from $\mathrm{P} / \mathrm{E}$ boundary sections in Egypt. Strata, Toulouse, 1, vol. 9, p. 113-115.

\section{ANNEXE}

Liste alphabétique des nannofossiles calcaires trouvés dans la présente étude et cités dans le texte : Biantholithus sparsus BRAMLETTE et MARTINI, 1964 Blackites creber (DEFLANDRE in DEFLANDRE et FERT, 1954) SHERWOOD, 1974

B. herculeus (STRADNER, 1969) AUBRY, 1999

B. solus (PERCH-Nielsen, 1971) AUBRY, 1999

B. truncatus (BRAMLETTE et SULLIVAN, 1961) VAROL, 1989

Bomolithus elegans ROTH, 1973

Braarudosphaera alta Romein, 1979

B. bigelowii (GRAN et BRAARUD, 1935) DEFLANDRE, 1947

Campylosphaera eodela BUKRY et Percival, 1971

Coccolithus crassus BRAMLETTE et SULLIVAN, 1961

Cruciplacolithus tenuis (STRADNER, 1961) HAY et MOHLER, 1967 in HAY et al., 1967

Discoaster cruciformis MARTINI, 1958

D. diastypus Bramlette et Sullivan, 1961

D. falcatus BRAMLETTE et SULLIVAN, 1961

D. lenticularis Bramlette et Sullivan, 1961

D. lodoensis BRAMLETTE et RIEDEL, 1954

D. mahmoudii PERCH-NIELSEN, 1981

D. mohleri BUKRY \& PERCIVAL, 1971

D. multiradiatus BRAMLETTE et RIEDEL, 1954

D. nobilis MARTINI, 1961

D. splendidus MARTINI, 1960

Discoasteroides bramlettei BUKRY et PERcIVAL, 1971

Ellipsolithus distichus (BRAMLETTE et SULLIVAN, 1961) SullivaN, 1964

E. macellus (BRAMLETTE et Sullivan, 1961) Sullivan, 1964

Ericsonia universa (WIND et WISE, 1977) RoMEIN, 1979

Fasciculithus alanii PERCH-NIELSEN, 1971

F. billii PERCH-Nielsen, 1971

F. janii PERCH-NIELSEN, 1971

F. pileatus BUKRY, 1973

F. schaubii HAY et MOHLER, 1967
F. sidereus ByBELL et SELF-TraIL, 1995

F. tympaniformis HAY et MOHLER in HAY et al., 1967

F. ulii PERCH-NIELSEN, 1971

Helicosphaera seminulum BRAMLETTE et SUlLIVAN, 1961

Heliolithus kleinpellii SUlLIVAN, 1964

H. knoxii STEURBAUT, 1998

H. megastypus (BRAMLETTE et Suldivan, 1961)

ROMEIN, 1979

$H$. riedelii Bramlette et SUllivan, 1961

Lophodolithus reniformis BRAMLETTE et SULLIVAN, 1961

Markalius inversus (DEFLANDRE in DEFLANDRE et FERT, 1954) BRAMLETTE et MARTINI, 1964

Micrantholithus inaequalis MARTINI, 1961

Micrantholithus spp.

Neochiastozygus digitosus PERCH-NIELSEN, 1971

N. distentus (Bramlette et Sullivan, 1961) Perch-

NIELSEN, 1971

Neococcolithes protenus (BRAMLETTE et SULLIVAN, 1961) BLACK, 1967

Neocrepidolithus spp.

Pentaster lisbonensis BYBELL et GARTNER, 1972

Pontosphaera spp.

Prinsius bisulcus (Stradner, 1961) HaY et MOHLER, 1967

Reticulofenestra spp.

Rhomboaster cuspis BRAMLETTE et SULLIVAN, 1961

Rhomboaster sp.

Scapholithus apertus HAY et MOHLER, 1967

Semihololithus biskayae PERCH-NIELSEN, 1971

S. kerabyi PERCH-NIELSEN, 1971

Sphenolithus primus PERCH-NIELSEN, 1971

Toweius eminens (BRAMLETTE et SULLIVAN, 1961)

Perch-Nielsen, 1971

T. gammation (BRAMLETTE et SulLIVAN, 1961) ROMEIN, 1979

T. pertusus (SUlLIVAN, 1965) RoMeIN, 1979

T, serotinus BYBELL et SELF-TRAIL, 1995

T. tovae PERCH-NIELSEN, 1971

Tribrachiatus orthostylus SHAMRAI, 1963

Trochoaster operosus (DEFLANDRE, 1954) MARTINI et STRADNER, 1960

Zygodiscus herlynii SULlIVAN, 1964

Zygrhablithus bijugatus (DEFLANDRE in DEFLANDRE et FERT, 1954) DEFLANDRE, 1959

Z. bijugatus nolfii STEURBAUT, 1991

Liste des espèces citées dans le texte, mais non retrouvées :

Discoaster anartios BYBELL et SELF-TraIL, 1995

D. araneus BUKRY, 1971

Rhomboaster calcitrapa GARTNER, 1971

Tribrachiatus bramlettei (BRÖNNIMANN et STRADNER, 1960) Proto Decima et al., 1975. 